\title{
Stability CONCEPts in Bargaining Games
}

by

\author{
Somnath Kundu \\ Master of Data Science, Ryerson University, Canada, 2017 \\ Master of Engineering, Sambalpur University, India, 1996 \\ Bachelor of Engineering, Calcutta University, India, 1991
}

\author{
A thesis \\ presented to Ryerson University \\ in partial fulfillment of the \\ requirements for the degree of \\ Master of Science \\ in the Program of \\ Applied Mathematics
}

Toronto, Ontario, Canada, 2019

(C)Somnath Kundu, 2019 


\section{AUTHOR'S DECLARATION FOR ELECTRONIC SUBMISSION OF A THESIS}

I hereby declare that I am the sole author of this thesis. This is a true copy of the thesis, including any required final revisions, as accepted by my examiners.

I authorize Ryerson University to lend this thesis to other institutions or individuals for the purpose of scholarly research.

I further authorize Ryerson University to reproduce this thesis by photocopying or by other means, in total or in part, at the request of other institutions or individuals for the purpose of scholarly research.

I understand that my thesis may be made electronically available to the public. 


\author{
Abstract \\ Master of Science 2019 \\ Somnath Kundu \\ Applied Mathematics \\ Ryerson University
}

Stability concepts in Bargaining Games

In this thesis we discuss some novel concepts of stability in bargaining games, over a network setting. So far, the studies on bargaining games were done as profit sharing problems, whose underlying combinatorial optimization problems are of packing type. In our work, we study bargaining games from a cost sharing perspective, where the underlying combinatorial optimization problems are covering type problems. Unlike previous studies, where bargaining processes are restricted to only two players, we study bargaining games over a more generic hypergraph setting, which allows any bargaining process to be formed among any number of players. In previous studies of bargaining games, the objects that are being negotiated are assumed to be uniform and only the outcomes of the negotiations are allowed to be different. However, in our study, we accommodate possibilities of non-uniform weights of the objects that are being negotiated, which is closer to any real life scenario. Finally we extend our study to incorporate socially aware players by introducing a relaxed and innovative definition of stability. 


\section{Acknowledgements}

I would first like to convey my deepest gratitude to my thesis supervisor Dr. Konstantionos Georgiou. Working with him on this thesis, has been one of the greatest experiences of my academic life. He always had confidence in me, pushed me and made me accomplish the work which I never thought, I would be able to do.

I would also like to thank all the professors and the academic staff of Ryerson Mathematics Department. They were warm, encouraging and always there at the time of need. It has been a wondeful experience studying in the Mathematics Department of Ryerson University.

Last but not least, I would also like to acknowledge all my fellow students. I am greatly indebted to them as I learnt a lot from each of them. 


\section{Contents}

Declaration

Abstract

Acknowledgements

Dedication $\quad$ v

1 Organization of the Thesis 1

2 Introduction $\quad 2$

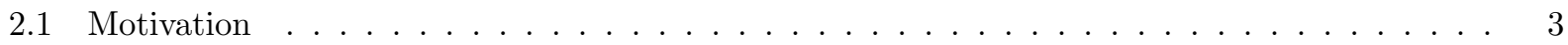

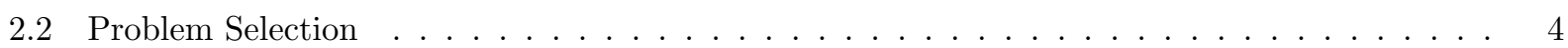

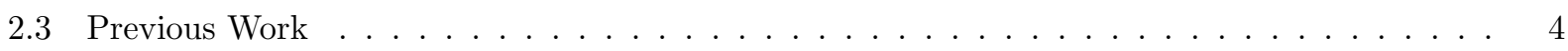

2.4 Our Contribution . . . . . . . . . . . . . . . . . . . . . 6

3 Salient Mathematical Concepts $\quad 7$

3.1 Graphs and Hypergraphs . . . . . . . . . . . . . . . . . . . . . . . 7

3.2 Game Theory Basics . . . . . . . . . . . . . . . . . . . . . . . 8

3.3 Bargaining Games . . . . . . . . . . . . . . . . . . . . . . . . . 10

3.4 Combinatorial Optimization . . . . . . . . . . . . . . . . . . . . 12

3.5 Linear Programming . . . . . . . . . . . . . . . . . . . . . . . . . . 13

3.6 Integer Programming . . . . . . . . . . . . . . . . . . . . . . . . . 14

3.7 Duality Theory . . . . . . . . . . . . . . . . . . . . . . . 17

3.8 Integrality Gap . . . . . . . . . . . . . . . . . . . . . . . . . . 18

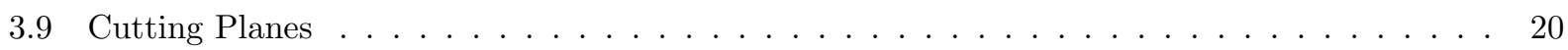

4 Main Work $\quad 2$

4.1 Cost sharing Bargaining Games . . . . . . . . . . . . . . . . . . . . . . . . . . . 22

4.2 Casting Bargaining Game as a Generic Game . . . . . . . . . . . . . . . . . . . . . 27

4.3 Stability Concept . . . . . . . . . . . . . . . . . . . . . . . 27 


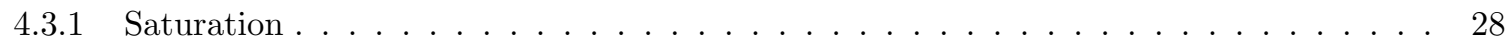

4.3.2 Arriving at a stability definition . . . . . . . . . . . . . . . . . 29

4.4 Relationship of Stability with Integrality Gap . . . . . . . . . . . . . . . . . . . . 31

4.4.1 Combinatorial Optimization Problem . . . . . . . . . . . . . . 31

4.4 .2 Integrality from Stability . . . . . . . . . . . . . . . . . . . . 32

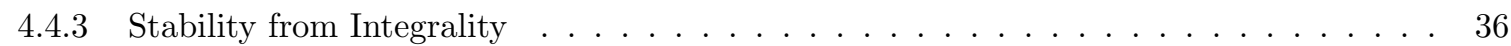

4.5 Stability for socially aware agents . . . . . . . . . . . . . . . . . . . . . . 41

4.5.1 Critical Constraints . . . . . . . . . . . . . . . . . . . 43

4.5.2 Combinatorial Optimization with the New Constraints . . . . . . . . . . . . . . 43

4.5.3 Relaxed Notion of Stability . . . . . . . . . . . . . . . . . . . . . . 43

4.5.4 Augmented Representation of the Bargaining Game . . . . . . . . . . . . . . . . 45

4.5.5 Augmented Game and the Revised Combinatorial Optimization Problem . . . . . . . 47

4.5.6 Relationship of the new Stability with Integrality Gap . . . . . . . . . . . . . . . . 48

5 Possible Future Work $\quad 5$

Bibliography
56 


\section{List of Figures}

3.1 Simple graph and Hypergraph . . . . . . . . . . . . . . . . . . . . . . . . 8

3.2 Solution concepts of a bargaining game . . . . . . . . . . . . . . . . . . . 11

3.3 Combinatorial Optimization Problem . . . . . . . . . . . . . . . . . . . . . 14

3.4 Linear Programing Problem . . . . . . . . . . . . . . . . . . . . . . . . . . 15

3.5 Integer Programing Problem _. . . . . . . . . . . . . . . . . . . . . 16

3.6 Cutting Plane . . . . . . . . . . . . . . . . . . . . . . . . 19

3.7 Cutting Plane . . . . . . . . . . . . . . . . . . . . . . 21

4.1 Bargaining Game Example with Hypergraph. . . . . . . . . . . . . . . . . . . . . . . . . 24

4.2 Bargaining Game Example with a Bipartite graph . . . . . . . . . . . . . . . . . . . . 25

4.3 Bargaining Game Example with Odd Cycle. . . . . . . . . . . . . . . . . . . . . . . . . . 42

4.4 Payment distribution with critical constraints for tight agent . . . . . . . . . . . . . 45

4.5 Payment distribution with critical constraints for oversaturated agent . . . . . . . . . . 46

4.6 Augmented representation of the game with critical constraints . . . . . . . . . . . . . 47 


\section{Chapter 1}

\section{Organization of the Thesis}

This thesis is divided into four main chapters. The first chapter (Chapter 2) is the introductory chapter where we describe mainly the motivation and the introduction of the problem. We also describe the previous work related to our work. In the next chapter (Chapter 3) we include some salient mathematical concepts that we used in our work. The next chapter (Chapter 4) is the part where we describe our work in complete detail. In the last chapter (Chapter 5) we describe the possible future work. 


\section{Chapter 2}

\section{Introduction}

Within a social network setting, individuals are engaged in a constant struggle to maximize their own interest. To achieve this feat, individuals often form coalitions among themselves and try to extract out maximum interest from those coalitions. Individuals weigh in their options and negotiate among themselves to collect the maximum benefit from different coalitions. These processes give rise to bargaining actions among players, which are studied under the realm of bargaining game theory. From the context of game theory, the term Game is used to describe a general collection of rules within which self-interested and rational individuals engage among themselves to maximize certain outcome for serving their own interest. Bargaining games are one type of game which mainly describes or formulates the rules necessary for bargaining processes among individuals in an isolated or an interconnected environment.

In any bargaining game there can be a large number of possible outcomes. Given that fact, it is of great importance to know if in a particular outcome every player is satisfied or some players could find a better arrangement outside of the outcome and want to disrupt the outcome. That means, one of the most pressing questions in the study of bargaining games is whether a bargaining game can attain a stable outcome or not. This question involves complicated analysis, given the fact that stability can be perceived in different ways by different persons. It is also possible that a bargaining game may not have a stable solution.

In our present work we study stability of bargaining games and its novel relationships with combinatorial optimization. We carry out our work of bargaining games from a cost sharing perspective, where players have certain demands that they need to fulfill by choosing different services. These services are generated by coalitions among the players, and the costs of those services are to be shared by the participating members. To the best of our knowledge, this is in contrast to all the previous studies of bargaining games, where the game is cast as a profit sharing game. We also generalize the study of bargaining game by allowing services to have any number of players, which needs to be described by a hypergraph. This is in contrast to the previous studies where a contract can only be formed by two players, which can be described by a simple graph. In addition to that we have introduced further complexity by introducing different satisfaction amounts for 
each player from each service. With this arrangement we define a notion of stability, which is intuitive and practically useful, yet is completely interrelated with the solution of the underlying combinatorial problem of the bargaining game. In our work we show this relationship of stability and the integrality gap (discussed later) of the corresponding relaxed linear program of the bargaining game. This formal proof is extremely profound from a practical sense as well. Because of this proof, we can be sure that a bargaining game can attain a stable outcome if we just solve the corresponding linear program and find an optimum integral solution of the linear program.

We also extend our stability definition to accommodate the case of socially aware agents as well. With our intuitive but strict definition of stability, it is possible that many of the bargaining games may never attain a stable solution. However, if we relax our notion of stability, then it is possible to show that in some more cases we can have stable solutions. In our work we formulate this amount of relaxation that is necessary for an outcome to be stable, by carefully crafting a relaxed notion of stability. With this new notion of stability we again prove the same relationship of stability and the integrality gap of the corresponding linear program.

\subsection{Motivation}

Consider a community or a village, where many people reside. The villagers or the members in the community know some other people around them inside that community. Each person needs different kinds and different amounts of services. For example, food, houses, clothing, etc. Assume that, it is a remote community and everyone needs to produce his or her service with assistance from other people around him/her. So persons can team up with the other persons to create those services. For example, some four or five people are needed to collectively cultivate and grow food. One person alone can not grow any food. For each of the service, members need to put in their time. It is imperative that every member would try to minimize their total time commitment and for that he/she would look around and see with whom to team up. For that reason, some alliances would be formed and people will come to an agreement as to who will spend how much time in which alliance. Now the question is, if they once come up with an arrangement between themselves, is it possible for some of them to find a better arrangement where they have to spend less time than their current arrangement. If that is the case, then they would most certainly want to deviate from their current arrangement. Is it possible that they will ever reach to an arrangement where no one could find a better solution? Or in other words, would they ever come to a stable arrangement? What criteria would define those stable conditions? Is there any way we can be sure that there exists a stable arrangement, or will some situations assert that the stable arrangement can never be found. These are the interesting questions we want to study mathematically in our current thesis work. 


\subsection{Problem Selection}

Bargaining games can be either profit sharing games or cost sharing games. The profit sharing games are categorized as Packing Type problems, where the individuals want to maximize their profit from different coalitions. It is to be noted that different coalitions generate different profits. This becomes challenging, because each individual can't engage in an infinite number of coalitions. Rather, each individual has a capacity or a maximum number of allowed coalitions. So the individuals need to choose their coalitions wisely in order to maximize their profit.

The cost sharing games are exact conjugate of the profit sharing games. The cost sharing games are categorized as Covering Type problems, where the individuals choose different services to fulfill their demands. Here the different services cost different amounts, and they would want to minimize the cost for different coalitions or services they are part of. So, similar to the profit sharing problem, individuals need to chose their services wisely in order to minimize their cost here as well.

Almost all the studies of bargaining games were done with the Packing type problem, but in our work we conduct our study as a covering type problem. As described before, almost all the studies of bargaining games were carried out with simple graphs, where the alliances can only be between two persons. But in our work we study bargaining game in hypergraphs, where alliances can happen between any number of persons. Another important aspect of our work is that we generalize the games by allowing outcomes of every alliance for every person to be different, which is not the case for previous work on bargaining games. In previous studies on bargaining games, it is considered that every person receives unit satisfaction from every alliance.

To our knowledge, we are the first to study stability of bargaining games in the context of socially aware members. There are often situations where within social settings, if everyone is extremely selfish, everyone gets stuck in a bottleneck situation where no one wins because of instability. But in those particular situations, if individuals are rational enough to be a little flexible then it is possible to arrive at stable arrangements which benefit everyone. Now the question would be how can we quantify how much relaxation is needed from each individual to make the situation stable, where no one would feel that there could have been a better solution for him or her. In our work we are going to investigate those situations in mathematical terms.

\subsection{Previous Work}

Bargaining games have been studied extensively in various disciplines, especially in Economics, Sociology and Management. In economics, bargaining games are studied mainly as cooperative games to understand the resource distributions, whereas in sociology bargaining games are studied mainly as Network Exchange Theory to understand the social dynamics. In management, bargaining games are studied mainly to strategise business directions. Many scholars and mathematicians have studied different aspects of Bargaining games. For example Rubinstein's work [27] extended the Nash equilibrium concept of bargaining games. 
However, in our study we focus on stability which is one of the solution concepts of bargaining game. We discuss how the study of stability aspects of bargaining games have been shaped over the years.

The study of equilibrium and fairness concepts in bargaining game theory first started with the work of John F. Nash . In his paper [24] he described a mathematical model for balanced bargaining outcomes. Consider a two player bargaining game where players $A$ and $B$ form a contract and generate a profit of 1 dollar. Player $A$ can get $\alpha \in[0,1]$ dollars and $B$ can get $\beta \in[0,1]$ dollars, respectively if they don't participate in the contract. That means player $A$ and $B$ has options $\alpha$ and $\beta$ dollar respectively outside of the contract. The surplus of the contract can be thought of as $1-(\alpha+\beta)$. Nash proposed that a fair outcome of the bargaining game is when the surplus is divided equally to each participant, which means that in the balanced outcome of the bargaining game $A$ and $B$ each receives $\frac{1}{2}(1-(\alpha+\beta))$. In bargaining game theory this model is referred as Nash Bargaining Solution.

The generalized version of Nash solution which gives rise to the concept of balanced solution was first introduced by Karen et al.[10] and Sharon C. Rochford [26] in two different papers. It is notable that the notion of balance also agrees very well with the experimental results as described by David Willer in his book "Network Exchange Theory" [30].

However our main focus of research is the stability concept in bargaining games. The basic foundation of our work is based on the work of Kleinberg and Tardos [21]. They studied bargaining games from the context of packing-type problems. In their analysis, each player (agent) can form only one contract with their neighbours within a network setting, which can be represented by a simple graph. They show that such games have balanced solutions if they have stable solutions. They also show another interesting property: that the existence of a stable solution is characterized by the integrality gap of a basic linear program relaxation for the associated combinatorial optimization problem. This work is the foundation stone of our work.

Later, Bateni et al. [2] extended the work of Kleinberg and Tardos to bipartite simple graphs. In their model they allow agents to have more than one contract. They also showed that stable outcomes correspond to allocations in the core (one solution concept of cooperative games, which somewhat relates to the equilibrium concept) of the underlying coalition game, thereby establishing a relationship between network bargaining and co-operative game theory. Network bargaining games were previously studied by many in the context of assignment games. Some notable mentions out of those lists are Shapely and Shubik [28], X. Deng et al. [12], Granot et al. [16], X. Deng and Q. Fang [11], Chalkiadakis et al. [8], etc. Farczadi et al. [14] extended the results of the Kleinberg-Tardos model for networks with agents with general capacities. Hajiaghayi et al. [18] also did the work in that line. Georgiou, et al. [15] considered again packing-type (matching) problems in which agents can bargain over a network from distance.

In recent years, different variations of bargaining games have been studied. Kanoria et.al. [20] studied network bargaining games with general capacities in natural dynamics. Some papers [1], [5], [13] studied 
local dynamics in network bargaining games . Tanmoy et al. [6] and [7] considered packing-type bargaining games with no capacity constraints, but with agents' utilities being nonlinear. Byati et al. [3] studied the dynamics of bargaining within a market context while considering a local dynamical model of a one-sided exchange network with transferable utilities. Huber Chan et al. [9], introduced alternative models for network bargaining, in which players themselves are the negotiated objects and had instances with no stable outcomes. Boch et al. [4] and Ito et.al. [19] studied the problem of minimally modifying the graph for injecting stability when bargaining instances do not admit stable solutions.

\subsection{Our Contribution}

In our work we build on top of the study of Kleinberg and Tardos [21], Bateni et al. [2] and Farczadi et al. [14]. The common thread between their work and ours is the study of stability over a network bargaining game. However in all of their work, the underlying problem of the bargaining game was of packing type, where the payoffs are distributed among the players. In contrast to that, in our case we are studying bargaining games where the costs are shared by the players and the players want to minimize their costs. This fact makes the problem a covering type problem. Our bargaining game model is more generic, as services are of non-uniform value, i.e. the same service may provide different satisfactions to different agents. In those previous studies contracts are between two players which are depicted by simple graph. However, in our study we allow a contract (service) to have more than two players, which means our bargaining games need to be depicted by hypergraphs. The inclusion of hypergraphs allow us to extend our work on stability for the socially aware agents. Using the concept of cutting plane for the underlying linear optimization problems, we introduce a relaxed notion of stability. This new relaxed notion of stability can make some games to have

stable outcome, which would otherwise be deemed as unstable, in a strict stability condition induced by the integrality gap of their underlying linear optimization problems. 


\section{Chapter 3}

\section{Salient Mathematical Concepts}

In this section we want to give the reader a gentle introduction about some of the mathematical concepts, that are used in our work. The major part of our work involves exploring the stability condition of outcomes of bargaining games and its relationship with combinatorial optimization concepts. We are going to describe these concepts in a little more detail. We are also going to touch on the definition of graphs and hypergraphs as our work involves bargaining games on a hypergraph.

\subsection{Graphs and Hypergraphs}

Graphs are mathematical structures which depict relationships between different objects. The objects are represented by the nodes of the graph and the relationships among them are described by the edges of the graph. So a graph is a tuple of two interrelated sets, one set is the set of all the vertices and another set is the relationships among those vertices. A relationship among different objects are defined as a set that contains all those objects. For example, if $A, B, C, D, E$ are nodes in a graph that represent different objects and the edges of the graphs are $\{A, B\},\{B, C, D\},\{A, E\}$, then $\{A, B\}$ indicates that there is a relationship between the objects $A$ and $B,\{B, C, D\}$ indicates that there is a relationship among the objects $B, C$ and $D$ and $\{A, E\}$ indicates that there is a relationship between the objects $A$ and $E$. If in a graph there are only two members in any of its edge then it is called a simple graph. If in a graph an edge has any number of nodes as its members then the graph is called hypergraph. So a hypergraph is a generalization of simple graph and the set of edges of a hypergraph is a subset of the power set of the nodes.

Mathematically, a graph $G$ is a tuple of two sets $V$ and $E$, where $V$ denotes the set of objects or nodes and $E$ denotes the set of edges among them. Simple Graph $G$ is given by $G=(V, E), \quad V \in\{1, \ldots, n\}, \quad n \in$ $\mathbf{N}, \quad E \subseteq\{\{i, j\}: i, j \in V, i \neq j\}$ and Hypergraph $G$ is given by $G=(V, E), \quad V \in\{1, \ldots, n\}, \quad n \in \mathbf{N}, \quad E \subseteq$ $2^{V} \backslash \emptyset$. See examples below. 


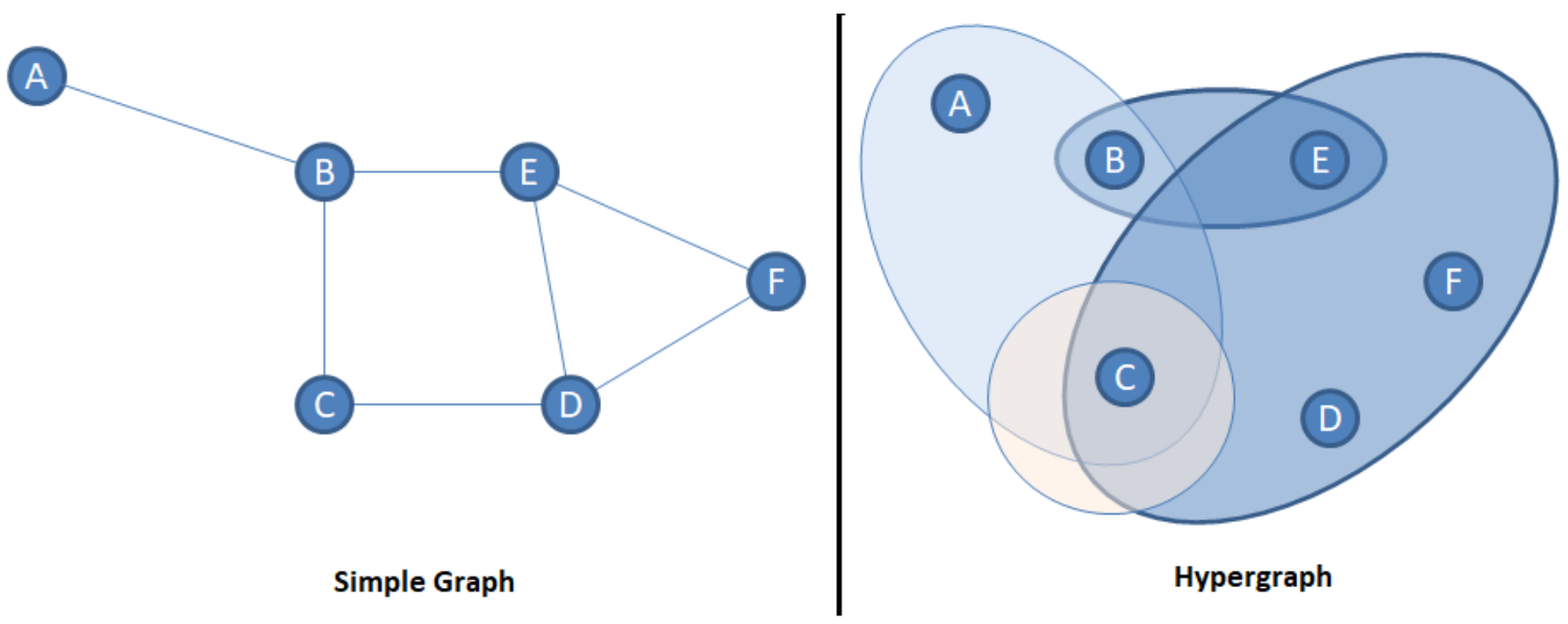

Figure 3.1: Simple graph and Hypergraph

\subsection{Game Theory Basics}

In Mathematics, Economics, Social Science, Management and in many other related disciplines, the term Game conceptually refers to the compilation of strategic interactions among decision makers to maximize their self interest. Game theory is the discipline which studies all the methods and formulations of those interactions. The basic assumptions of Game theory are that the decision makers are rational. An individual is called rational, when she picks the best possible choices for herself out of different possible actions by weighing out the relative merits of all possible actions. In general, any game has the following components :

1. Set of Decision makers, whom we call Players or Agents. We call it as $V$.

2. Set of all possible actions of all the players. We denote $A_{i}$ to be the set of possible actions for every player $i \in V$.

3. Functions that describe the relative consequences of all possible actions for every player. We denote $A$ as the Cartesian product of all the action sets of all the players. So $A=\times_{i \in V} A_{i}$. Here we define function $U_{i}$ for every player $i \in V$, such that $U_{i}: A \longmapsto \mathbb{R}$.

Considering all these points discussed above the generic definition of a game is given as follows :

Definition 3.2.1. Any Game $G$ can be defined as a tuple, given by $G=\left(V=\{1, \ldots, n\},\left\{A_{i}\right\}_{i \in V},\left\{U_{i}\right\}_{i \in V}\right)$ where $n \in \mathbb{N}, A=\times_{i \in V} A_{i}$ and $U_{i}: A \longmapsto \mathbb{R}$.

The third component, i.e., the relative consequences are called the utility functions. From a very naive sense it can be said that these utility functions collectively define the rules of the Game. Within the structure of those possible actions and the utility functions of the game, the players would try to maximize their own interests. Here an important point to be noted is that we are describing the quantification of self-interest of a player as utility and not using words like profit, because it is possible that the players would want to help 
the society, instead of just acquiring profit for themselves. In that case, the self interest of the players or the utility of the players is the social service. The word utility can also be used in terms of cost. In that case maximizing utility would inherently mean minimizing cost. In a game, players act based on the decisions they think are best for themselves, which generate a particular outcome of that game. The players could find different decisions, which they might think are best for them. The combinations of all those decisions of all those players can generate different sets of outcomes. Most of the studies in game theory revolve around the effort of finding which outcomes are best (or fair) or stable (or in equilibrium) for all players. There are many different ways the notion of best or stable outcome of a game can be defined. This different notion of fairness or stability in a game give rise to many different interesting formulations of game theory, which are useful for analyzing and predicting many real life problems in our society, business, government affairs, etc. Let us understand the concept of equilibrium in games using an example.

Example 3.2.1. Consider a game with two players $A$ and $B$. Player $A$ has two possible options $O_{A 1}$ and $O_{A 2}$ and Player $B$ has two possible options $O_{B 1}$ and $O_{B 2}$.

The utility or pay-offs for the player $A$ is given in the following table:

\begin{tabular}{|c||c|c|}
\hline \multicolumn{1}{|r||}{${ }^{A}$} & $O_{A 1}$ & $O_{A 2}$ \\
\hline \hline$O_{B 1}$ & 2 & 3 \\
\hline$O_{B 2}$ & 0 & 1 \\
\hline
\end{tabular}

The utility or pay-offs for the player $B$ is given in the following table:

\begin{tabular}{|c||c|c|}
\hline \multicolumn{1}{|c||}{${ }^{A}$} & $O_{A 1}$ & $O_{A 2}$ \\
\hline \hline$O_{B 1}$ & 2 & 0 \\
\hline$O_{B 2}$ & 3 & 1 \\
\hline
\end{tabular}

If player $A$ selects the first option $\left(O_{A 1}\right)$ then he gets 2 , if $B$ also selects the first option $\left(O_{B 1}\right)$ and gets nothing if $B$ selects the second option $\left(O_{B 2}\right)$. If $A$ selects the second option $\left(O_{A 2}\right)$ then he would get 3 if $B$ selects the first option $\left(O_{B 1}\right)$ and will get 1 if $B$ also selects the second option $\left(O_{B 2}\right)$. Considering all the choices, it is clear that the second option $\left(O_{A 2}\right)$ is the most logical choice for the player A. So A will play the second choice. For the exact same reason player $B$ will also play the second choice. So the outcome of this game is always going to end up as $A$ and $B$ both playing the second choice. This game is then going to attain an equilibrium, where none of the players would have any logical incentive to deviate from the outcome $\left(O_{A 1}, O_{B 2}\right)$ that we just deduced.

The above mentioned example is a slight variation of a famous game theory problem called "Prisoner's Dilemma" and the above mentioned equilibrium is an example of so called "Nash Equilibrium". It is to be noted that in many games "Nash Equilibrium" is not attained. 


\subsection{Bargaining Games}

Bargaining Game is a type of multi-player game, where players form alliances with each other and generate some utilities (not to be confused by the utility as described before). These utilities are then shared among the players. As discussed before, those utilities can also be expressed in terms of cost. In any bargaining game the following components are present. (This is not the formal definition of bargaining game. We define the bargaining game formally later in the thesis).

1. Set of Players : This indicates the collection of all the players who are playing the bargaining game. The players are generally denoted by a natural number index $i \in \mathbb{N}$. The set of players $V$ is given by $V=\{1, \ldots, n\}, n \in \mathbb{N}$. If a bargaining game is depicted by a Graph, then the nodes or the vertices of the graph generally represent the players.

2. Set of possible alliances: This indicates the set of alliances that members can form among themselves. Thus every alliance is just a subset of the players. So an alliance $e$ is given by $e \subseteq V$. The set of all the possible alliances $E$ is given by $E=\{e: e \subseteq V\}$. In the study of bargaining games it is often found that the alliances are allowed only between two players. However, in general sense, an alliance can happen among any number of players. So the set is actually a subset of the power set of the players, without the degenerate set, i.e, $E \subseteq 2^{V} \backslash \emptyset$. If in a graph the players are represented by the nodes then the edges represent the possible alliances among them.

3. Set of utilities from the alliances : Every alliance will generate some sort of utilities which the members will share among them. Based on the type of game this utility can be profit (for packing type problems) or cost (for covering type problems) for the members. This utility is denoted by $\left\{C_{e}\right\}_{e \in E}$. If in a graph the edges represent the alliances, then the edge weights represent the corresponding utilities of the alliances.

4. Set of constraints for the players : Every player has some sort of constraint amount that need to be satisfied. Depending on the type of bargaining games, these constraints can be either capacity (for packing type problems) or demand (for covering type problems) of the players. The set of all those constraint values for each player is denoted by $\left\{D_{i}\right\}_{i \in V}$. If in a graph the nodes represent the players, then the node weights represent the corresponding demand or capacity of the player.

As mentioned above, in a bargaining game, players form alliances with each other to maximize the utility for them. During this process, players need to make sure that their demands or capacities are satisfied. They analyze all their options and form only the alliances that best suit their interest. Each player negotiates with others to obtain as much as utilities for herself. They are always on the lookout for getting a better deal, which means they would always try to find better utilities for themselves outside of their present alliances. With this dynamic process every bargaining game generates different outcomes. The outcome of a bargaining game has the following components.

1. Set of possible alliances: This indicates the set of alliances that members have formed among themselves. As indicated before, all available alliances are not guaranteed to be materialized, only a 
subset of the alliances will be materialized. The set of possible alliances can be denoted by $A$, which is given by $A \subseteq E$.

2. Set of utility share : This indicates how the utility of each alliances are distributed among each player. This can be denoted by $\left\{S_{i, e}\right\}_{i \in V, e \in E}$.

Now, if an outcome satisfies all the constraints (in this case either demand or capacity) of a bargaining game then that is called a feasible outcome. If a feasible outcome of a bargaining game is such that no players could increase their utility by individually deviating from the current outcome, then this particular outcome is called a stable outcome. This notion of stability can be defined in various ways, but in general it is intuitive that if any player receives more utility from an alliance outside of their materialized contract, then she will let go of the contract where she is currently in and take the new contract. This concept is the very foundation of any stability analysis of a bargaining game. In this context it is to be noted that the balanced outcome of a bargaining game is a stricter concept than stability. According to Nash [24], the balanced outcome can be thought of as the equal division of the surplus utility of an alliance after each members' outside options are deducted from the generated utility.

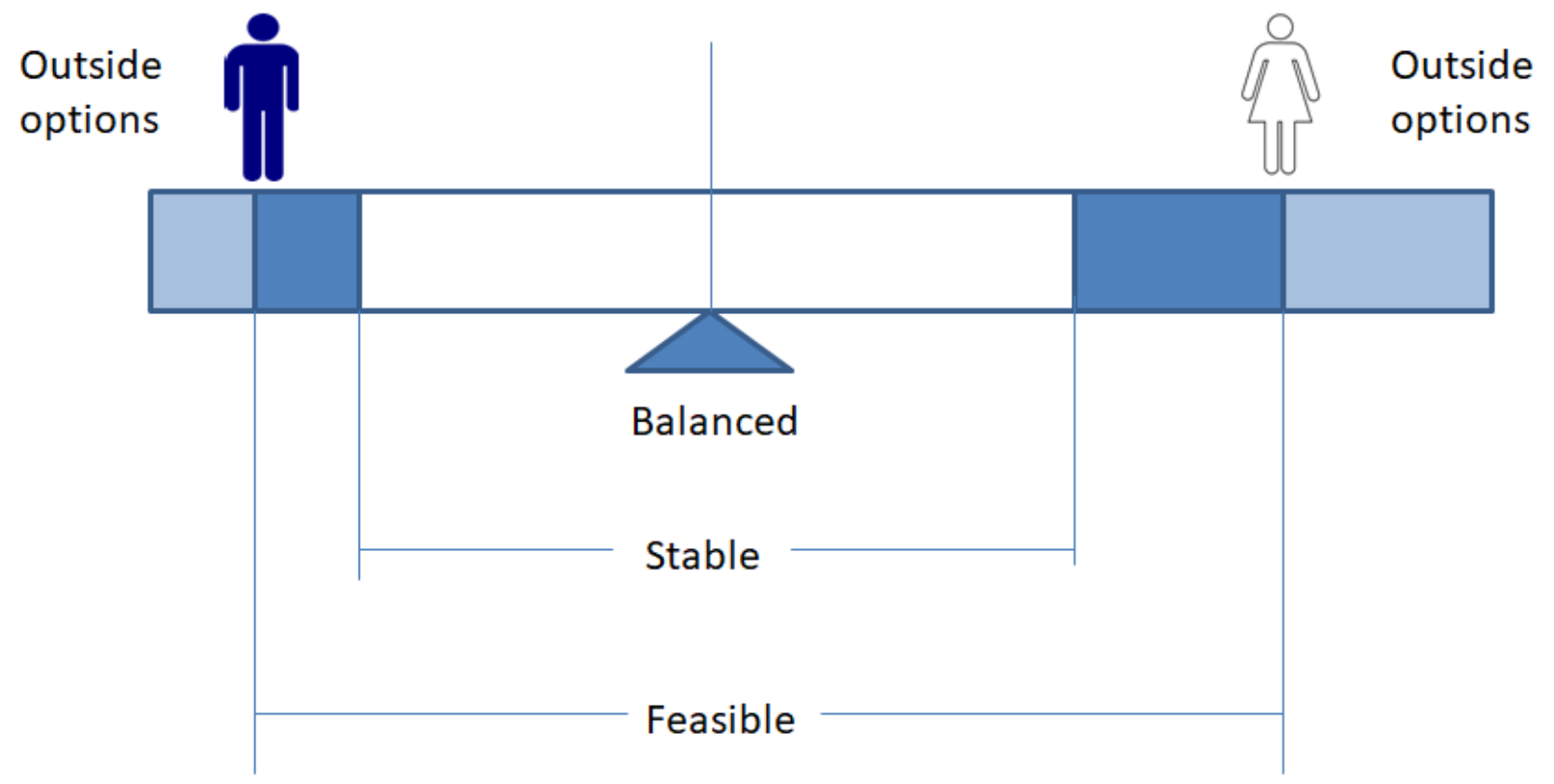

Figure 3.2: Solution concepts of a bargaining game

Example 3.3.1. Figure [3.2] describes the notion of stability and balanced outcome schematically. Consider in a bargaining game, there are two players $A$ and $B$. They form an alliance which generates profit of $P$. Suppose that $A$ doesn't take this contract then he will get $\alpha \in \mathbb{R}_{+}$and if $B$ doesn't take this contract then she will get $\beta \in \mathbb{R}_{+}$. Assume $A$ and $B$ would split the profit $P$ between them and they would get $S_{A}$ and $S_{B}$ respectively. Any arrangement of $S_{A}, S_{B} \in \mathbb{R}_{+}$which makes $S_{A}+S_{B}=P$, would be a feasible outcome of 
the game. But if $A$ or $B$ get less than what they would have gotten outside of this contract, they would want to come out of this contract and take what they would get outside of it. That means if $S_{A}<\alpha$ or $S_{B}<\beta$, the outcome won't be stable. In other words the outcome would be stable if $S_{A} \geq \alpha$ and $S_{B} \geq \beta$; The outcome also has to be feasible for it to be stable, i.e, $S_{A}+S_{B}=P$. So there can be multiple arrangements of $S_{A}$ and $S_{B}$ which will satisfy the stability conditions $\left(S_{A}+S_{B}=P, S_{A} \geq \alpha, S_{B} \geq \beta\right)$. That means there can be many stable outcomes of this game. Out of all those outcomes, we can find a pair of $S_{A}$ and $S_{B}$ such that $S_{A}=\alpha+\frac{1}{2}(P-(\alpha+\beta))$ and $S_{B}=\beta+\frac{1}{2}(P-(\alpha+\beta))$. In this case at first, the possible amounts outside of the alliances are given to the players $A$ and $B$, then the total amounts outside of the alliance $(\alpha+\beta)$ is deducted from the total profit and the surplus amount is equally divided between the players. This arrangement is going to be termed as the balanced outcome of the game.

In our work, we study the stability concept of bargaining games extensively. Our work involves bargaining games which are of covering type problem. In future chapter, we formally define the bargaining game that we are going to study, but here we define a typical example of a covering type bargaining game and its solution concepts for the benefit of the readers.

Definition 3.3.1. A covering type bargaining game $B$ in a simple graph with unit satisfaction is a tuple. $B=\left(G=(V=\{1, \ldots, n\}, E \subseteq\{\{i, j\}: i, j \in V, i \neq j\}),\left\{C_{e} \in \mathbf{R}_{+}\right\}_{e \in E},\left\{D_{i} \in \mathbf{R}_{+}\right\}_{i \in V}\right)$ where $n \in \mathbf{N}$.

Definition 3.3.2. Given a covering type bargaining game $B$ in a simple graph with unit satisfaction an outcome $F$ of the game is defined as a tuple. $F=\left(A \subseteq E,\left\{S_{i, e} \in \mathbf{R}_{+}\right\}_{i \in V, e \in E}\right)$

This is just given here to formally introduce the reader with a formal definition of bargaining game as a covering type problem. This definition is not used anywhere else in our work. We have defined the bargaining game that we study formally later in our work.

\subsection{Combinatorial Optimization}

Combinatorial optimization is a study of finding best or optimum options out of different finite possible options. Different possible options are determined by some rules or a set of constraints and the best option is determined by an objective function. The goal of a Combinatorial Optimization problem is to maximize or minimize the objective function, while satisfying all the constraints. Most of the combinatorial optimization problems can be formulated mathematically. Every mathematical formulation of a combinatorial optimization problem involves a set of variables. Each variable indicates different aspects of the optimization problem. The objective function and the constraints involve those variables. The constraints are a set of equalities and inequalities involving those variables. An example of a typical mathematical formulation of a combinatorial optimization is as follows.

$$
\begin{array}{r}
\min f(x): \\
g(x) \geq b \\
h(x) \geq c \\
x \in \mathbb{R}_{+}{ }^{n}
\end{array}
$$




$$
\begin{gathered}
f, g, h: \mathbb{R}_{+}{ }^{n} \longmapsto \mathbb{R}_{+} \\
b, c \in \mathbb{R}
\end{gathered}
$$

Many combinatorial optimization problems fall under the umbrella of two broad categories, called Packing Type problems and Covering Type problems. The objective of Packing Type problems is to maximize the objective function within the constraints of " $\leq$ " inequalities. In case of Covering Type problems, the objective is to minimize the objective function with the " $\geq$ " inequality constraints. Using the appropriate use of sign, it is possible to express any combinatorial optimization problem as minimization problem and any constraint can be expressed as a " $\leq$ " inequalities

The feasible solution of a combinatorial optimization problem is a set of values of the variables for which the constraints are satisfied. The combinations of all the feasible solutions of a combinatorial optimization forms a feasible solution space for that combinatorial optimization. The optimum solutions of a combinatorial optimization are some solutions from its feasible solution space for which the objective function attains its extreme (maximum or minimum depending on the problem) value. The optimal value of a combinatorial optimization problem is the value that the objective function generates with the values of the optimum solutions. It is possible to have different optimal solutions of a combinatorial optimization problem which gives the same optimal value for that optimization problem. Figure [3.3] illustrates the conceptual view of how the variables, feasible solution space and the objective functions fit together in the context of a Mathematical Program.

In this context, one point to be noted is that if the feasible solution space is convex and the objective function is convex, then for a minimization problem any local minimum optimum solution is guaranteed to be a global minimum optimum solution. So the convex feasible solution space and the convex objective function makes it easier to solve a combinatorial optimization problem.

\subsection{Linear Programming}

If in a Mathematical Program, the objective function and all the constraint functions are linear, then the formulation of the problem is called linear programming. Linear programming problems have convex feasible solution space. This feasible solution space of a linear program which is bounded by different linear hyperplanes (represent constraints) are called "Polyhedra". Linear programming problems can be solved in polynomial time (under mild assumptions); (Eg. using Ellipsoid method). Figure [3.4] gives a pictorial representation of a Linear Programming problem, its feasible region and the objective function. An example of a typical linear programming is as follows.

$$
\begin{aligned}
& \min c^{T} x: \\
& A x \geq b \\
& x \geq 0
\end{aligned}
$$




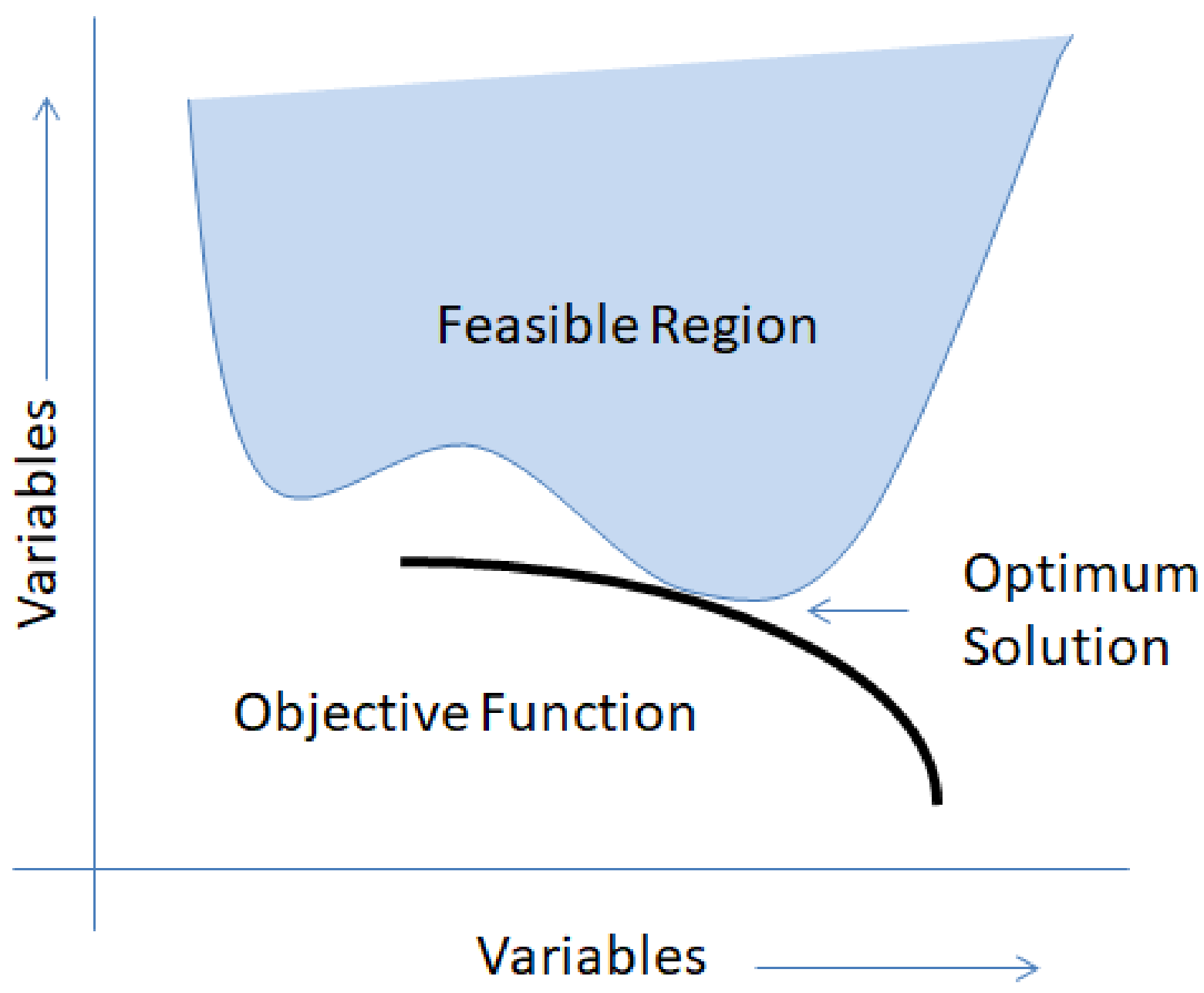

Figure 3.3: Combinatorial Optimization Problem

$$
A \in \mathbb{R}^{m \times n}, \quad b, c \in \mathbb{R}^{n}
$$

\subsection{Integer Programming}

If in a mathematical program, the objective function and all the constraint functions are linear and also all the variables are required to be integers, then the formulation of that combinatorial optimization is called Integer Programming. The formulation of the Integer programming is very similar to the Linear programming, except for the fact that all the variables have to be integers. Figure [3.5] demonstrates an example of an Integer Program in comparison to a linear program. An example of a typical integer 


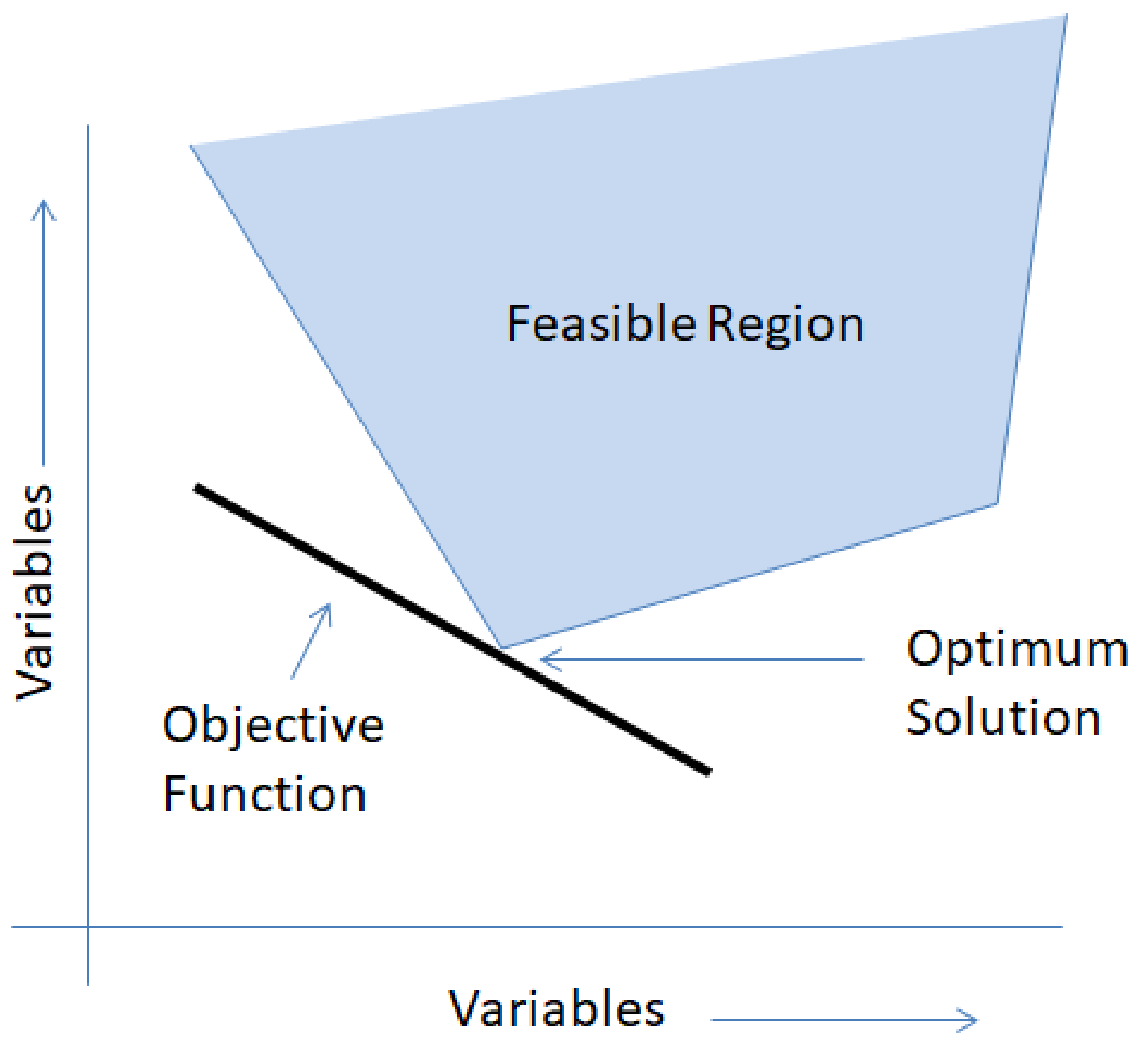

Figure 3.4: Linear Programing Problem 
programming formulation is as follows.

$$
\begin{gathered}
\min c^{T} x: \\
A x \geq b \\
x \in \mathbb{Z}^{n} \\
A \in \mathbb{R}^{m \times n}, \quad b, c \in \mathbb{R}^{n}
\end{gathered}
$$

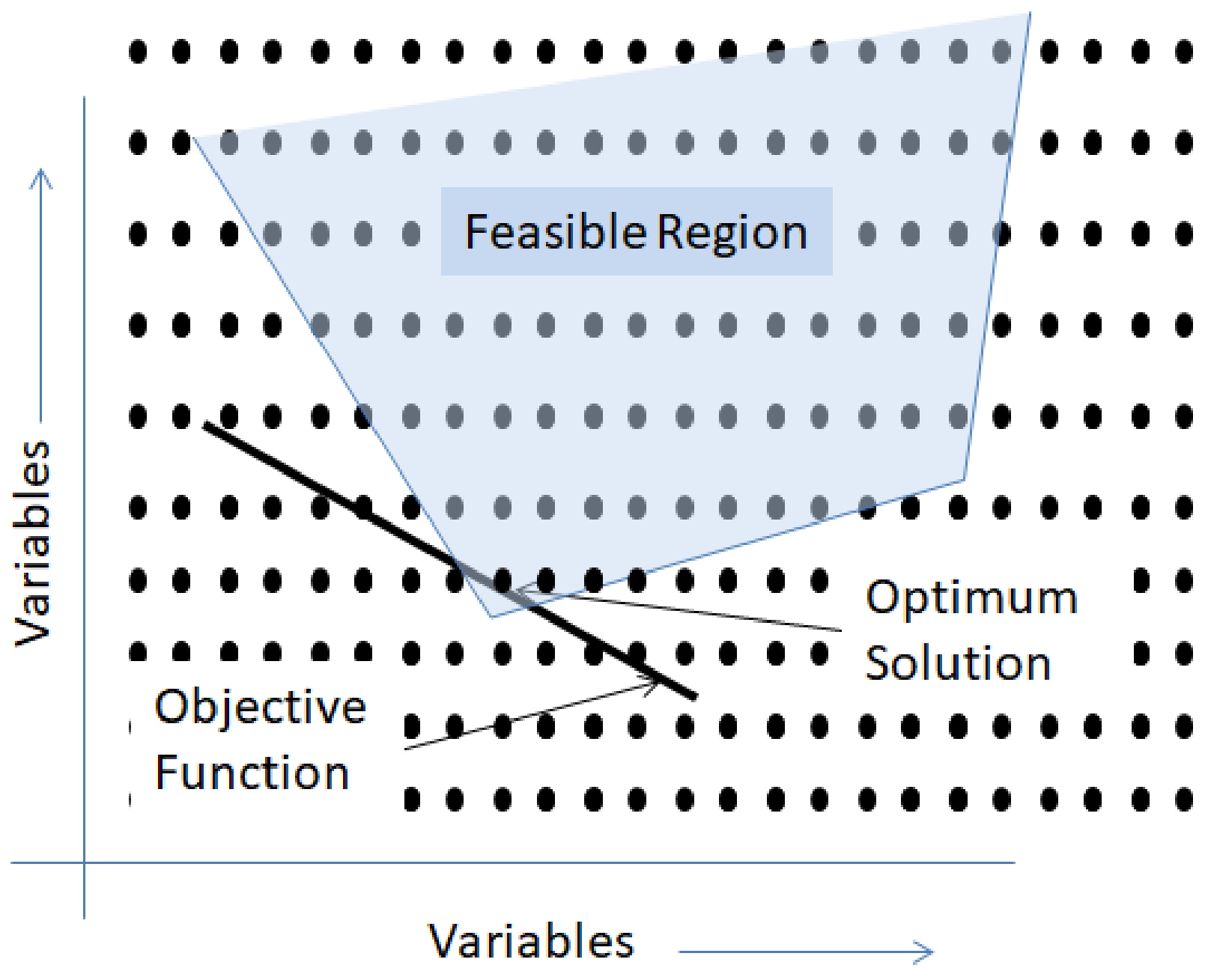

Figure 3.5: Integer Programing Problem 


\subsection{Duality Theory}

Every Linear Programming (LP) formulation has one corresponding complementary linear programming formulation called dual. If the original (we call it the "primal") problem is a minimization problem, then its dual is a maximization problem and vice versa. The variables of primal problem have bijective relation with the constraints of the dual and the constraints of the primal have bijective relation with the variables of the dual. It can be proved formally that the dual of a dual is the original primal problem. Consider a Primal LP, with $A \in \mathbb{R}^{m \times n}, b \in \mathbb{R}^{m}, c \in \mathbb{R}^{n}$

$$
\begin{aligned}
\min c^{T} x & \\
A x & \geq b \\
x & \geq 0
\end{aligned}
$$

The corresponding dual of the above LP is given by

$$
\begin{array}{r}
\max b^{T} y: \\
A^{T} y \leq c \\
y \geq 0
\end{array}
$$

Suppose the optimum solutions of above primal and dual LP exists and they are $x_{0}$ and $y_{0}$ respectively, then the optimal values for them will be given by $c^{T} x_{0}$ and $b^{T} y_{0}$. The duality gap is defined as $\left(c^{T} x_{0}-b^{T} y_{0}\right)$. This duality gap is always non-negative. If the duality gap is 0 then this condition is called a strong duality. If the duality gap is non-negative then this condition is called a weak duality. As per the theorem of (von Neumann [1947], Gale, Kuhn and Tucker [1951]) for any linear problem, if both the primal and dual LP have feasible solutions, then it can be proved that the strong duality holds for the optimum solution and the weak duality theory holds for any feasible solution.

From the above mentioned theorem it can be proven that, with $A \in \mathbb{R}^{m \times n}, b \in \mathbb{R}^{m}, c \in \mathbb{R}^{n}, \min \left\{c^{T} x\right.$ : $\left.A x \geq b, x \in \mathbb{R}_{+}{ }^{n}\right\}$ and $\max \left\{b^{T} y: A y \leq c, y \in \mathbb{R}_{+}{ }^{m}\right\}$ are two primal and dual pair, then $x_{0}$ and $y_{0}$ are the feasible solutions to the primal and dual LP, if and only if

$$
\begin{array}{r}
x_{0} \cdot\left(c-A^{T} y_{0}\right)=0 \\
y_{0} \cdot\left(b-A x_{0}\right)=0
\end{array}
$$

[Here the $\cdot$ indicates "dot" products between the vectors]

These above conditions are called contemporary slackness condition. 


\subsection{Integrality Gap}

Integer programming problems are not convex, more specifically their solution space is not a convex set. They are NP-Hard problems. It is often the practice that the Integer programming problems are relaxed so that the variables are allowed to have non-integer real values. This way the relaxed program becomes a linear programming problem, which can be solved in polynomial time. It is possible that the actual optimal integer solution does not coincide with the solution of its relaxed linear programming solution. The deviation of these two solutions are measured by Integrality Gap. The definition of integrality gap of the relaxed linear program for a integer program is given as follows:

Definition 3.8.1. Consider a particular instance $C$ of a combinatorial optimization problem. An exact formulation is given by $I P(C)$ and its corresponding relaxed linear formulation is given by $L P(C)$. The optimum value for those are given by $\operatorname{opt}_{I P}(C)$ and $\operatorname{opt}_{L P}(C)$ respectively. If the combinatorial optimization problem is a minimization problem then the integrality gap $I G_{C}$ of $L P(C)$ for the problem $C$ is defined as:

$$
I G_{C}=\inf _{C}\left(\frac{o p t_{L P}(C)}{o p t_{I P}(C)}\right)
$$

If the combinatorial optimization problem is a maximization problem then the integrality gap $I G_{C}$ of $L P(C)$ for the problem $C$ is defined as :

$$
I G_{C}=\sup _{C}\left(\frac{o p t_{I P}(C)}{o p t_{L P}(C)}\right)
$$

From the above definition it follows that $I G_{C} \in[0,1]$, i.e., the integrality gap is a real number between 0 and 1. If for a particular optimization problem there is an integral optimum solution then the integarilty gap is 1. Figure [3.6] demonstrates the relaxed linear program of an integer program and the deviation between them.

Example 3.8.1. Consider a vertex cover problem for a simple graph $G=(V, E)$, where $G$ is the set of vertices and $E$ is the set of edges. For this problem we need to find the smallest subset $S \subseteq V$ of the vertices such that the union of all the neighbouring edges of all the modes of that particular subset is the set of all edges in the graph. In another way we say that at least one endpoint of every edge of the graph should be found in that subset. We can formulate this optimization problem in the following way.

Fix indicator variables $x_{i}$ for every vertex $i \in V, x_{i}=1$, if $i \in S$, else 0 . Formulation is given by:

$$
\begin{array}{r}
\min \sum_{i \in V} x_{i}: \\
x_{i}+x_{j} \geq 1 \quad \forall i j \in E \\
x_{i} \in\{0,1\} \quad \forall i \in V
\end{array}
$$


The above fomrulation is IP. If we want to relax the formulation the corresponding LP would be given by:

$$
\begin{array}{r}
\min \sum_{i \in V} x_{i}: \\
x_{i}+x_{j} \geq 1 \quad \forall i j \in E \\
x_{i} \in[0,1] \quad \forall i \in V
\end{array}
$$

If we take a complete simple graph with 3 vertices, the graph will be a triangle. It follows that the exact optimum value for this vertex cover problem would be 2. But as per the LP, if we take $x_{i}=\frac{1}{2}, \forall i \in V$ then the optimum value for the relaxed LP becomes $\frac{3}{2}$. In this case the integerality gap becomes $\frac{3 / 2}{2}$ or $\frac{3}{4}$.

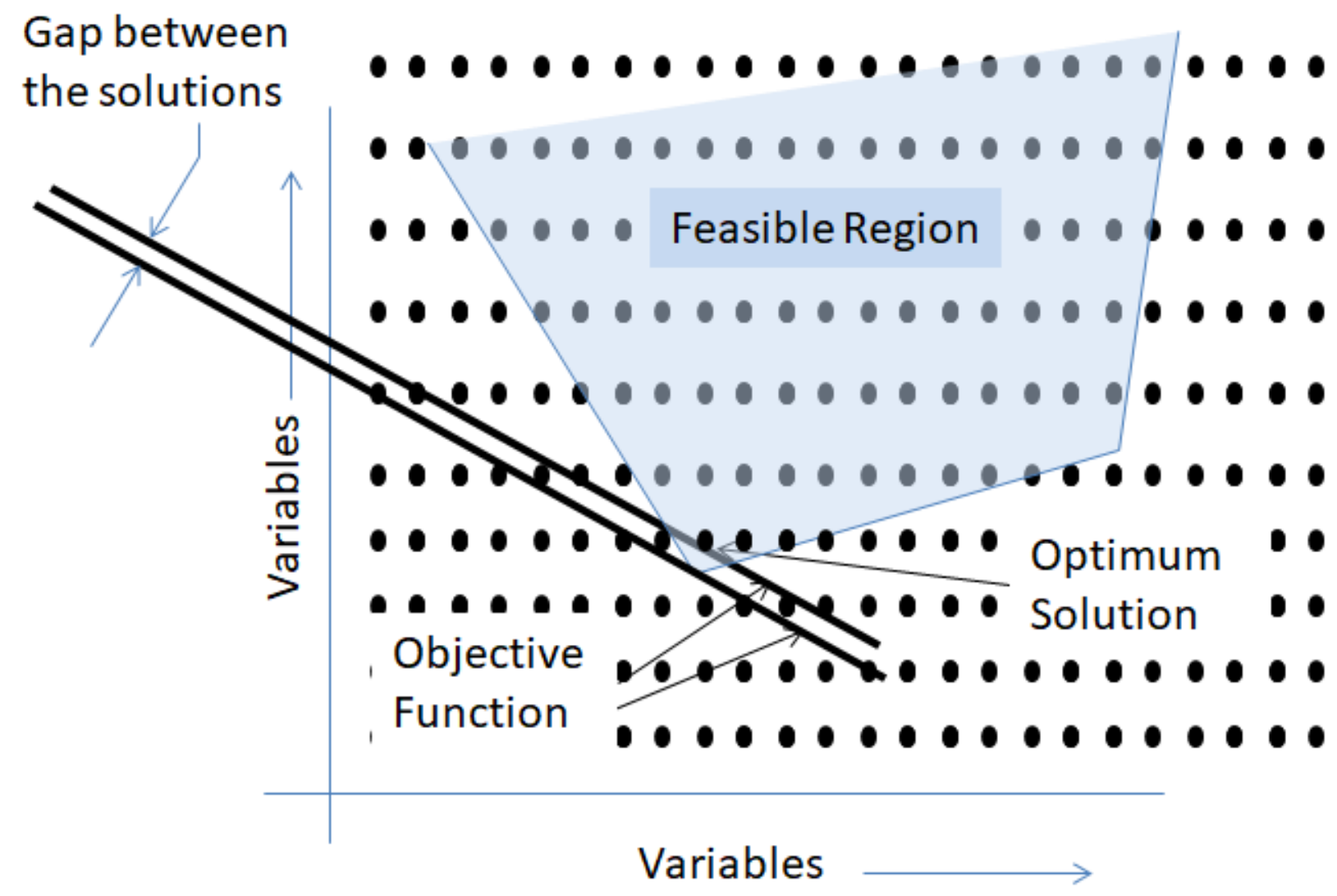

Figure 3.6: Cutting Plane 


\subsection{Cutting Planes}

From the above discussion it is clear that if we relax the integer problem, it can be solved in polynomial time, but the solution it generates may deviate from the exact solution. In order to get around the problem an interesting approach is adopted which is called cutting planes technique. The idea of this is to introduce more linear constraints which will force the solutions to be integers. These constraints will be redundant in case of an integer program, but those are not redundant for the relaxed linear program. These extra set of constraints tighten the feasibility space of the relaxed linear programing. This can be thought of as those extra constraints are removing away the areas from the relaxed feasible regions, which are away from the integral hull (Integral hull is defined as the convex hull of the integer points in the feasible region of the relaxed linear program). That's the reason those extra set of constraints are aptly called cutting planes. See [Fig : 3.7] for an illustration of cutting plane.

Example 3.9.1. Consider the vertex cover problem as mentioned above for a simple complete graph with three vertices $G=(V, E), V=\{1,2,3\}, E=\{\{12\},\{23\},\{31\}\}$. The relaxed LP formulation is:

$$
\begin{array}{r}
\min x_{1}+x_{2}+x_{3}: \\
x_{1}+x_{2} \geq 1 \\
x_{2}+x_{3} \geq 1 \\
x_{3}+x_{1} \geq 1 \\
x_{1}, x_{2}, x_{3} \in[0,1]
\end{array}
$$

This gives the optimum value as $\frac{3}{2}$ and the integerality gap as $\frac{3}{4}$. If we add one extra linear constraint $x_{1}+x_{2}+x_{3} \geq 2$, then the linear program becomes the following

$$
\begin{array}{r}
\min x_{1}+x_{2}+x_{3}: \\
x_{1}+x_{2} \geq 1 \\
x_{2}+x_{3} \geq 1 \\
x_{3}+x_{1} \geq 1 \\
x_{1}+x_{2}+x_{3} \geq 2 \\
x_{1}, x_{2}, x_{3} \in[0,1]
\end{array}
$$

This revised LP gives the optimum solution as 2, which is same as the exact integral solution, i.e., the integrality gap becomes 1 . This extra constraint will be only satisfied if the solution is integral. This extra linear constraint doesn't alter any of the combinatorial optimization problem criteria, though this criteria is completely redundant for integer problem formulation. However, as we have seen, for the corresponding relaxed linear problem, this extra linear constraint tightens the relaxation and makes the solution integral. This way we can still use the linear program which can be solved in polynomial time yet will have the chance to get the exact integral solution or at least be closer to the exact solution. 


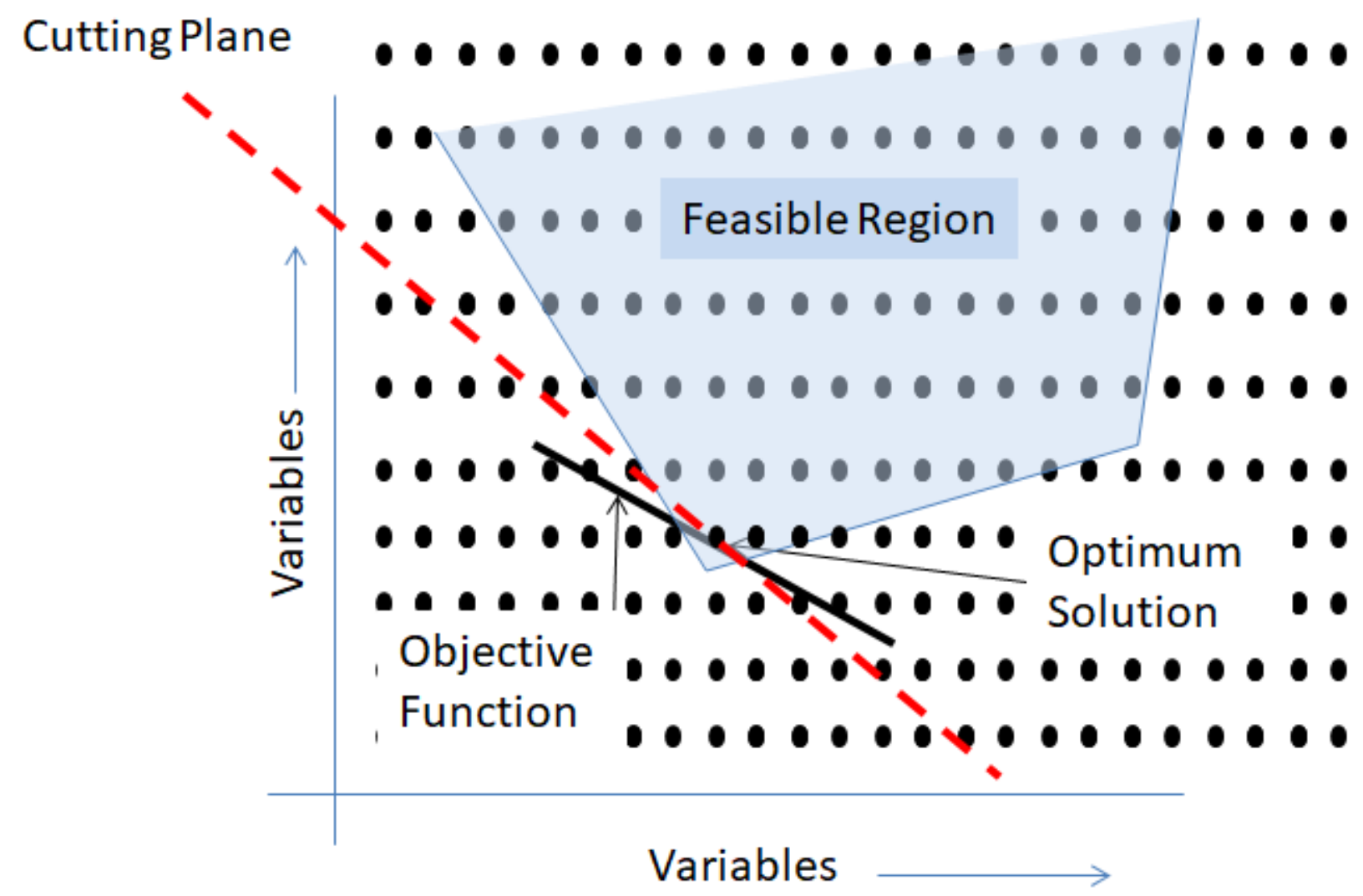

Figure 3.7: Cutting Plane

For better understanding about different topics on combinatorial optimization, linear programming, duality theory, integer programming, cutting planes, etc readers can consult any book on combinatorial optimization. Example, "A Gentle Introduction to Optimization" by B.Guenin, J. Konemann, L. Tuncel. [17] One example of book For the topics on game theory is "A Course in Game Theory" by Martin J. Osborne and Ariel Rubinstein [25]. 


\section{Chapter 4}

\section{Main Work}

Most of the studies of bargaining games were mainly confined within the domain of simple graphs, and those studies are mostly based on the payoff distribution of profits. These type of problems are considered to be packing problems. In contrast to that, we are going to study bargaining games as covering problems and in a more generic hypergraph setting. Also, we are going to study bargaining games where players can get different satisfaction from different contracts. This is more generic compared to the most frequently studied bargaining games where each contract provides just one unit of satisfaction to each player. Finally, we are going to study different concepts of stability notion which could accommodate intuitive behavior of socially aware agents.

\subsection{Cost sharing Bargaining Games}

For any bargaining game in a network setting, nodes represent players or agents, and edges represent possible contracts among those agents. In most of the previously studied bargaining games, each contract could only be formed between two agents. So simple graphs were sufficient to depict those type of bargaining games. We are trying to generalize bargaining games where contracts can be formed among any number of agents. To describe those generic games, we need to use hypergraphs where the edges can have any number of nodes.

Let us understand the bargaining game with a practical example.

Example 4.1.1. Consider a community that consists of 5 members and denote those members as $A, B, C, D, E$. This community encourages volunteering and social engagements. As a part of their social responsibility, each of them has to fill up a quota of volunteering points. Members $A, B, C, D, E$ have a quota of $5.1,4.0,5.0,3.5,5.2$ respectively. The community needs different types of projects, but not everyone is skilled enough to do all the projects. Different people are required to be teamed up for finishing different projects. Every project needs some time to be completed. This project time needs to be shared by the participating members of that project. It is to be noted that not all the projects are required to be completed, but each member must obtain her quota of the volunteering points. So, every member must select the projects she 
needs to complete her quota. Note that every member doesn't need to choose all the projects they are eligible to do. The possible projects and their eligible members are given as follows:

\begin{tabular}{|c|c|c|}
\hline No. & Time Required & Members \\
\hline 1 & 12.3 & $A, B, E$ \\
\hline 2 & 7.5 & $B, D$ \\
\hline 3 & 11.0 & $B, C$ \\
\hline 4 & 8.7 & $A, C$ \\
\hline 5 & 5.9 & $B, C, E$ \\
\hline 6 & 5.9 & $D, E$ \\
\hline
\end{tabular}

Different types of projects give different volunteering points to each participating members. They are given in the tabular form:

\begin{tabular}{|c|c|c|c|c|c|}
\hline No. & $A$ & $B$ & $C$ & $D$ & $E$ \\
\hline 1 & 2.35 & 1.75 & & & 1.80 \\
\hline 2 & & 1.20 & & 1.25 & \\
\hline 3 & & 2.05 & 2.40 & & \\
\hline 4 & 2.75 & & 3.00 & & \\
\hline 5 & & 1.05 & 2.27 & & 2.00 \\
\hline 6 & & & & 2.25 & 2.00 \\
\hline
\end{tabular}

In this scenario, each member would choose to select only the projects that would fulfill her quota of points, yet at the same time would minimize her time requirement. In this process, they would bargain among themselves as to who would give how much time for each of their participating project. Based on these collective negotiations of the members, some projects would be chosen by the members and some projects would be left unchosen.

This whole process can be studied as a bargaining game. Here we could have an underlying hypergraph, where the nodes are members or players and the edges are the projects, which can be thought of as services. The volunteering points needed by each member can be thought of as their demand, which are represented by the corresponding node weights. The time requirement of each project can be thought of as the cost per services, which is represented by the edge weight. The volunteering points that each member gets from each project can be thought of as her satisfaction, which are represented by a tabular form. See [Fig : 4.1]

Using the above example we could generalize and define the concept of bargaining games. Each bargaining game has some players or agents. The agents have their demand that they need to acquire from some services. Services are formed by different agents. Each service can have a different set of agents, and each agent can get a particular amount of satisfaction from a particular service. The agents would choose some services from their set of eligible services and collect the satisfactions from each of the chosen service. For each agent, the sum of all the satisfaction gathered from her participating services should be at least her demand. Each service costs some amount which needs to be shared by the agents. That means each service will cost some amount for each of the players. The players will try to minimize their overall cost while they 


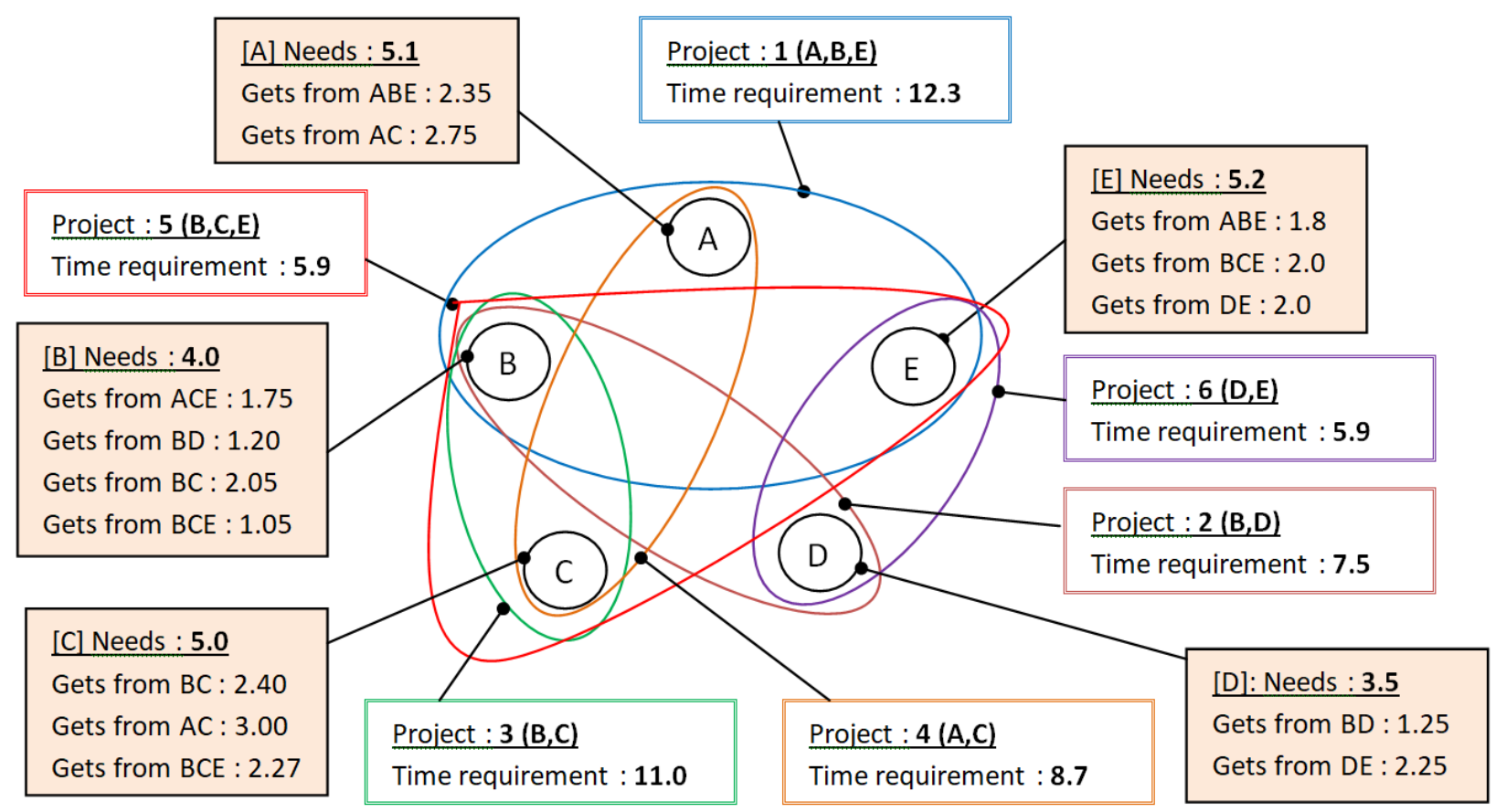

Figure 4.1: Bargaining Game Example with Hypergraph.

try to fulfill their individual demand. In that process they will choose some services out of all their possible choices and bargain to spend as little as possible, along other agents with whom she shares her services.

Bargaining games can be modeled on a network or a graph. The nodes of the graph would represent the agents, and the node weights would represent their corresponding demand. Here, each service can have any number of players. For this, we need to consider hypergraphs instead of simple graphs. The edges of the hypergraph would denote the possible services and the nodes of each edge will denote the participating agents of that service. Each edge weight will denote the amount, that each service costs. The satisfaction of each agents from different services can be given in a tabular form. It is to be noted that the hypergraph can be also represented as a simple bipartite graph, where the edges are depicted as nodes. See [Fig : 4.2]

If we want to describe the above mentioned bargaining game, in a more formal mathematical way, it can be defined as follows:

Definition 4.1.1. A bargaining game $B$ is determined by the following:

1. Hypergraph $\left.G=(V, E), \quad V \in\{1, \ldots, n\}, \quad n \in \mathbf{N}, \quad E \subseteq 2^{V} \backslash \emptyset\right\}$,

2. Edge weights $\left\{C_{e} \in \mathbf{R}_{+}\right\}_{e \in E}$

3. Vertex weights $\left\{D_{i} \in \mathbf{Q}_{+}\right\}_{i \in V}$ 


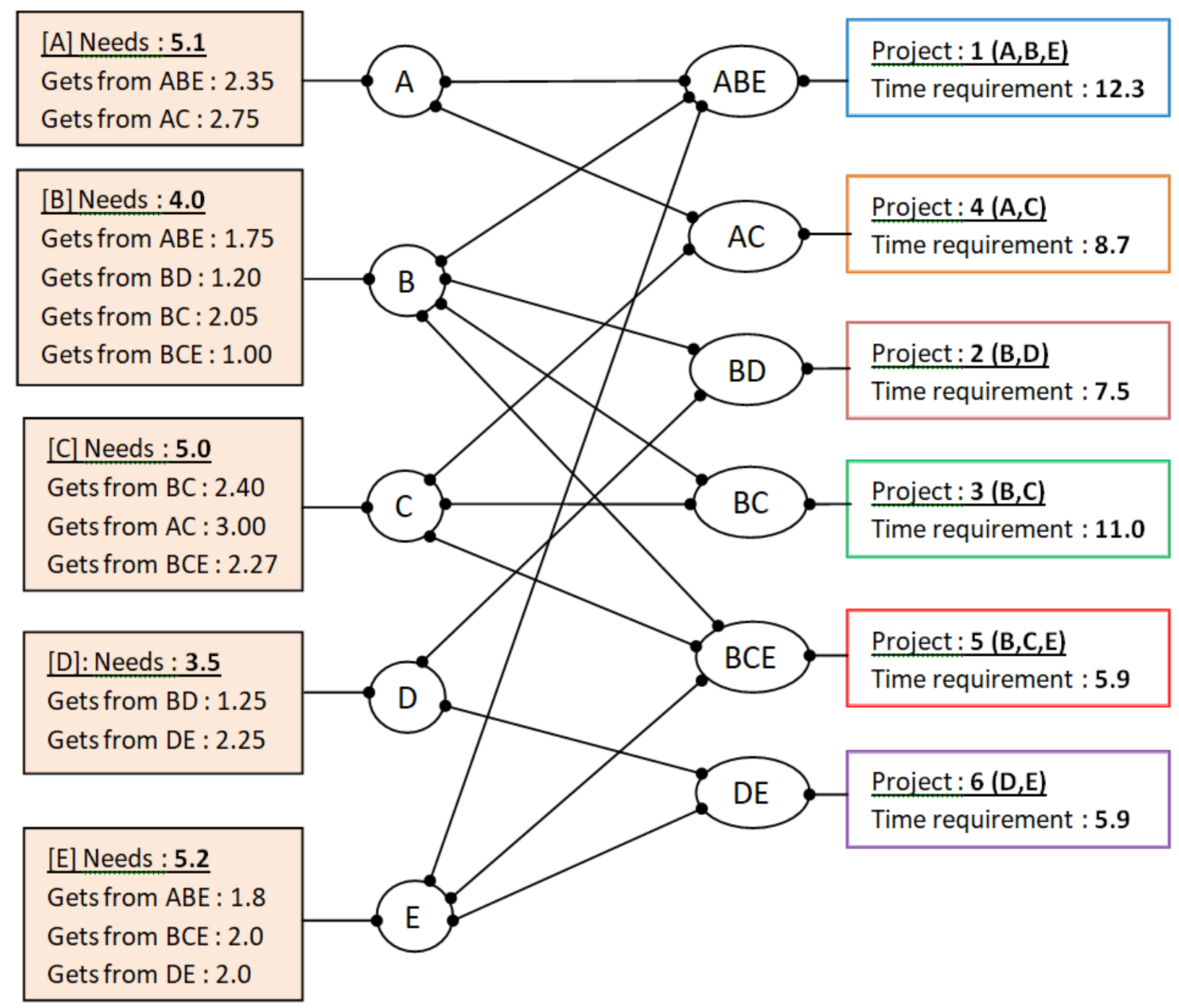

Figure 4.2: Bargaining Game Example a with a Bipartite graph 
4. Satisfaction of every player from every service $\left\{\rho_{i, e} \in \mathbf{Q}_{+}\right\}_{e \in E, i \in V}$

Notation : In this context, we also define the following terms, given hypergraph $G=(V, E)$

1. Edges containing a node $i \in V$ within a subgraph $G^{\prime}=(V, A) \quad A \subseteq E$ $\delta_{A}(i)=\{e \in A: i \in e\}$,

2. Neighbours of a node $i \in V$ within a subgraph $G^{\prime}=(V, A) \quad A \subseteq E$ $N_{A}(i)=\{m \in V: \exists e \in A, \quad(i, m) \in e\}$,

3. sign: $\mathbf{R}_{+} \longmapsto\{0,1\}$ :

$$
\operatorname{sign}(i)=\left\{\begin{array}{ll}
0 & \text { if } i=0 \\
1 & \text { if } i>0
\end{array} \quad i \in \mathbf{R}_{+}\right.
$$

An outcome of a bargaining game (defined above) is the set of services (edges) that are selected and the set of payments each player makes for each service. Mathematically it can be defined as follows:

Definition 4.1.2. Outcome $F$ of a bargaining game $B$ consists of the following :

1. Subset of the edges those are selected $A \subseteq E$

2. Payments of every player for every edge $\left\{S_{i, e} \in \mathbf{R}_{+}\right\}_{e \in E, i \in V}$

Definition 4.1.3. A feasible outcome $F=\left(A \subseteq E,\left\{S_{i, e} \in \mathbf{R}_{+}\right\}_{e \in E, i \in V}\right)$ is an outcome which satisfies the following two conditions :

1. All services are paid for : $\sum_{i \in V} S_{i, e}=C_{e} \quad \forall e \in A$

2. Every player meets her demand: $\sum_{e \in \delta_{A}(i)} \rho_{i, e} \geq D_{i} \quad \forall i \in V$

Example 4.1.2. Consider the bargaining game mentioned in example 1, then one of the feasible outcomes could be as follows :

Projects $A B E, B D, A C, B C E, D E$ are chosen. Project $B C$ is not chosen. If we check the total volunteering time obtained by each player

\begin{tabular}{|c|c|c|c|c|c|c|}
\hline Projects & Remarks & $A$ & $B$ & $C$ & $D$ & $E$ \\
\hline$A B E$ & Chosen & 2.35 & 1.75 & & & 1.80 \\
\hline$B D$ & Chosen & & 1.20 & & 1.25 & \\
\hline$B C$ & $\underline{\text { Not chosen }}$ & & $\underline{2.05}$ & $\underline{2.40}$ & & \\
\hline$A C$ & Chosen & 2.75 & & 3.00 & & \\
\hline$B C E$ & Chosen & & 1.05 & 2.27 & & 2.00 \\
\hline$D E$ & Chosen & & & & 2.25 & 2.00 \\
\hline Total & $\mathbf{5}$ out of $\mathbf{6}$ & $\mathbf{5 . 1}$ & $\mathbf{4 . 0}$ & $\mathbf{5 . 2 7}$ & $\mathbf{3 . 5}$ & $\mathbf{5 . 8}$ \\
\hline Requirement & & 5.1 & 4.0 & 5.0 & 3.5 & 5.2 \\
\hline
\end{tabular}


In this case every player fulfills the quota of her volunteering points.

Another component of the outcome of the bargaining game is how much time every player spends for what project. The following table shows that information:

\begin{tabular}{|c|c|c|c|c|c|c|}
\hline Projects & Time needed & $A$ & $B$ & $C$ & $D$ & $E$ \\
\hline$A B E$ & $\mathbf{1 2 . 3}$ & 6.15 & 0.00 & & & 6.15 \\
\hline$B D$ & $\mathbf{7 . 5}$ & & 0.00 & & 7.50 & \\
\hline$B C$ & $\underline{11.0}$ & & $\underline{0}$ & $\underline{0}$ & & \\
\hline$A C$ & $\mathbf{8 . 7}$ & 8.70 & & 0.00 & & \\
\hline$B C E$ & $\mathbf{5 . 9}$ & & 0.00 & 0.00 & & 5.90 \\
\hline$D E$ & $\mathbf{5 . 9}$ & & & & 2.95 & 2.95 \\
\hline
\end{tabular}

This means in this outcome of the bargaining game time requirements of every selected project is shared by the members. So as per our definition of feasibility, this outcome is feasible.

\subsection{Casting Bargaining Game as a Generic Game}

Any bargaining game can be thought as a special type of game. That means every bargaining game must have three components of a game, i.e. the players, their possible actions and the utility each player would get from all different combinations of possible actions. However, from the above mentioned definition isolating those three generic components of a game is not very intuitive. The bargaining game can be cast as a generic game the following way :

1. Set of Players : $V$

2. Actions for any player is a vector indexed by edges. That means each coordinate of the vector indicate how much that player pays for each edge. So the Action set is given by : $\left\{A_{i} \subseteq \mathbb{R}^{|E|}\right\}_{i \in V}$.

3. Utilities are defined as follows : Once players have selected a strategy (means they have decided on payments for each edge), then take all the edges whose costs are covered by the players' chosen payments. If those edges combinedly do not satisfy the demand of a particular player then that players utility is $-\infty$. If the demand of a particular player is satisfied then her utility is negative of sum of her payments.

\subsection{Stability Concept}

A stable outcome of a bargaining game is a particular feasible outcome where no agent has incentive to deviate from any of her current chosen contracts. That means in a stable outcome whatever total amount an agent is paying, for her current set of services, can not be greater than the total amount she might pay for any other possible set of services (other than her current set) she might choose to fulfill her demand. 
Another intuitive aspect of stability is that the agents would never want to pay for the services that they are not part of. Though in some special situation this may not be always true. We would discuss those special cases later. However, for the base concept of stability, agents do not pay for the services, they are not part of. This implies, that only the participating agents of a service pay for that service.

In order to explore stability in bargaining games, we need to study a concept called Saturation. Saturation can be thought of as a flag (a boolean state) which indicates if any agent, in a particular feasible outcome, is getting the satisfactions more than her demand or just tightly meet her demand.

\subsubsection{Saturation}

In any outcome of a bargaining game, if an agent is getting satisfactions more than her demand, then that agent is called an oversaturated agent. Intuitively, oversaturated agents are in a better position to bargain with the other agents who are tight. So, oversaturated agents are of particular importance for the analysis of stability in any bargaining game outcome.

Definition 4.3.1. Oversaturated agents $i \in V$ of a bargaining game $B$ with feasible outcome $F=(A \subseteq$ $\left.E,\left\{S_{i, e} \in \mathbf{R}_{+}\right\}_{e \in E, i \in V}\right)$ are those for which $\sum_{e \in \delta_{A}(i)} \rho_{i, e}>D_{i}$. The tight agents $j$ are those, who are not oversaturated, i.e tight agents $j$ are those, for which $\sum_{e \in \delta_{A}(j)} \rho_{j, e}=D_{j}$.

The set of all tight agents in an edge is given by $e^{T}=\left\{j \in e: \sum_{l \in \delta_{E}(j)} \rho_{j, l}=D_{j}\right\}$

Let us understand this concept with an example.

Example 4.3.1. Consider a bargaining game $B$ in a Hypergraph $G=(V, E)$. In that an agent $i \in V$ has possible services (edges) $e_{1}, e_{2}, e_{3} \in E$. The agent can get satisfactions $10,12,15$, from the edges respectively. Agent $i$ has demand 21. In one outcome of the bargaining game, three of those contracts $e_{1}, e_{2}, e_{3}$ are chosen. For simplicity, let us assume all the other agents who are also involved in the contracts $e_{1}, e_{2}, e_{3}$, are all tight and no agent has any other unchosen edges.

In the above scenario, agent $i$ is oversaturated; because, the total satisfaction, she is getting is $10+12+15=$ 37, which is more than her demand 21. If she drops any of the contracts, her demand would be still fulfilled. So she could offer to pay nothing for any of the service and others have to accept it. Because the others have tight condition, they can not loose her, as they need all of their current services where, she is required.

If we change the numbers a little bit, say her demand is 26 , then she can not loose the contract $e_{3}$. This will lower her negotiation power. If her demand is 36 , then even if she is saturated she can't loose any contracts. These concepts are intuitive and logical. But in our stability definition we would give more power to the oversaturated agents than they might actually have in practice. This concept of giving more power to the oversaturated agents, than they might actually have in practice, is important for our analytical proof and it guarantees that stability will not be violated. We will discuss this part in detail in the later chapter. 


\subsubsection{Arriving at a stability definition}

From the above example, it follows that if oversaturated agents can let go any of their contracts and still meet their demand, then they don't have to pay anything for any of their contracts. So if for any $f \in \delta_{E}(i)$ such that $\sum_{e \in \delta_{E}(i) \backslash f} \rho_{i, e} \geq D_{i}$ then agent $i$ would pay nothing for any of her services. That means $S_{i, e}=0$ for all $e \in \delta_{E}(i)$ if for any $f \in \delta_{E}(i)$ we find $\sum_{e \in \delta_{E}(i) \backslash f} \rho_{i, e} \geq D_{i}$

However, we are assuming absolute power for oversaturated agents in our definition of stability, i.e, every oversaturated agent pays nothing for any of her services. This arrangement doesn't conflict with the practical notion of stability, because in a bargaining game, if we give more negotiating power to some players, than they might actually have, then it is guaranteed that those players would never want to deviate from that arrangement; which means the arrangement will surely be stable for those players. We will find it later, that this condition is important to prove some of our proposed interesting theorem.

It is possible that, in practice, some of the oversaturated agents are not powerful enough to leave any of their contracts; yet in this condition they would not pay anything. With this arrangement, those people are still not going to deviate from this arrangement or deviate the stability condition. Here in this stability condition those oversaturated agents are favored little bit more than they should be, but still this condition guarantees the intuitive notion of stability.

The scenario is different for tight agents. Tight agents would be on the lookout to to replace any of their costly service with another unrealized service which might cost less for them. Here one point to be noted, that one agent might want to take an unrealized contract, but that wish doesn't guarantee that she would get that service. For any unrealized service, all the members of that service must have proper incentives to deviate from one of their existing services and benefit from taking this new service.

Consider an unrealized service (edge) is $f \in E \backslash A$, which has cost of $C_{f}$. A member of that service is denoted by $j \in f$. The satisfaction $j$ will get from that edge is given by $\rho_{j, f}$. The proposed cost that she may have to pay for this new service is given by $S_{j, f}$. So clearly, $\sum_{k \in f} S_{k, f}=C_{f}$.

Assume, that member $j$ already has a service $e: e \in \delta_{A}(j)$. The satisfaction the agent will get from that existing service are given by $\rho_{j, e}$. The amount the agent is paying for that existing services is given by $S_{j, e}$. The satisfaction per unit of cost the agent is getting from an existing service is then given by $\frac{S_{j, e}}{\rho_{j, e}}$. Agent must get at least that same satisfaction per unit of cost from the new contract, otherwise she has no incentive to deviate from the existing contract.

However for stability, if we require that each and every individual player needs to have a better option to deviate from the existing condition, then that would be too strict, because every other member of that unchosen edge has to agree. To analyze that, let us consider an edge $f \in E \backslash A$ which is not chosen in a particular outcome $\left(A \subseteq E,\left\{S_{i, e}\right\}_{i \in V, e \in V}\right)$. Every member $j \in f$ of that unchosen edge $f$ would be already 
part of some contracts $\delta_{A}(j)$ which are chosen in the outcome. Now we could take any one exiting contract $l_{j} \in \delta_{A}(j)$ of each member, who are part of that unchosen edge $f$, and add up the cost they are paying $S_{j, l_{j}}$ adjusted by a factor $\frac{\rho_{j, f}}{\rho_{j, l_{j}}}$, which basically means this adjustment factor is the ratio of the satisfaction of the member $j$, obtained from her proposed contracts $f$ and the existing contracts $l_{j}$. If we find that the summed up amount $\sum_{j \in f} \frac{\rho_{j, f}}{\rho_{j, l_{j}}} S_{j, l_{j}}$ is less than the total cost from the unchosen edge $\sum_{j \in f} S_{j, f}$, then the members won't want to loose their existing contract and take up the unchosen edge (contract). Thus the system would be stable.

We can conclude that for the outcome to be stable, the following conditions must be satisfied. $\forall e_{i} \in \delta_{A}(i)$, i.e., if we take any existing edge of each members of an unchosen edge, then

$$
\begin{aligned}
\sum_{j \in f} \frac{S_{j, e_{j}}}{\rho_{j, e_{j}}} \rho_{j, f} & \leq \sum_{j \in f} S_{j, f} \\
\rightarrow & \sum_{j \in f} \frac{S_{j, e_{j}}}{\rho_{j, e_{j}}} \rho_{j, f} \leq C_{f} .
\end{aligned}
$$

It is logical to think that if any player has to chose one contract to drop, among their existing contracts, she would want to let go of the contract that is costing her maximum. Because it is true for any existing edge of a particular member of an unchosen edge, we could write the condition as :

$$
\sum_{j \in f} \max _{e \in \delta_{A}(j)}\left(\frac{S_{j, e}}{\rho_{j, e}}\right) \rho_{j, f} \leq C_{f}
$$

Considering all those above mentioned conditions, the stability concept is defined as follows :

Definition 4.3.2. A feasible outcome $\left(A \subseteq E,\left\{S_{i, e}\right\}_{i \in V, e \in V}\right)$ of bargaining game $B$ would be stable if the outcome satisfies the following conditions :

1. No agent pays for the services that they are not part of : $S_{i, e}=0, \quad \forall e \notin \delta_{A}(i)$.

2. Power for oversaturated agents are considered : $S_{i, e}=0 \quad \forall e \in \delta_{A}(i): \sum_{e \in \delta_{A}(i)} \rho_{i, e}>D_{i}$.

3. Current outcome is the best outcome : $\sum_{j \in f} \max _{e \in \delta_{A}(j)}\left(\frac{S_{j, e}}{\rho_{j, e}}\right) \rho_{j, f} \leq C_{f} \quad \forall f \in E \backslash A$.

From the first condition it follows that :

$$
\sum_{i \in e} S_{i, e}=C_{e} \quad \forall e \in A
$$

This means, that only the members of a service $e \in A$ collectively pay for that service $e$ and no one else.

From the second condition it follows that :

$$
\left(\sum_{e \in \delta_{A}(i)} \rho_{i, e}-D_{i}\right) S_{i, e}=0 \quad \forall i \in V \quad \forall e \in E .
$$


This means, that only the tight agents pay positive amount for any service and oversaturated agents pays nothing for any of the service.

It is to be noted that in all practical purposes, the outcome is guaranteed to be stable if it satisfies all the conditions as defined in the above mentioned stability definition. But if the conditions are not satisfied then it is still possible for the outcome to be perceived as stable, from other intuitive point of view. So, from any practical purpose the above mentioned definition can be thought of as a sufficient condition of stability and not a Necessary condition of stability.

\subsection{Relationship of Stability with Integrality Gap}

\subsubsection{Combinatorial Optimization Problem}

Every bargaining game has an underlying combinatorial optimization problem. For this, we have to think from the perspective of the system as a whole and not from the individual perspective. Given a hypergraph $G=(V, E)$ (which represent the network where the game is conducted, here the nodes $V$ represent the players and the edges $E$ represent the possible services among the players) with edge weights $\left\{C_{e} \in \mathbf{R}_{+}\right\}_{e \in E}$ (which represent the costs of the services for all $e \in E$ ) and node weights $D_{i} \in \mathbf{N}$ (which represents the demands for players for all $i \in V)$. Satisfactions of players are given by $\left\{\rho_{i, e} \in \mathbf{Q}_{+}\right\}_{e \in E, i \in V}$. The objective for the combinatorial optimization problem is to minimize the overall cost of all the chosen services while making sure the demand of each player is fulfilled.

The above combinatorial optimization problem can be formulated as follows :

$$
\begin{array}{r}
\min \sum_{e \in E} C_{e} x_{e} \\
\sum_{e \in \delta_{E}(i)} \rho_{i, e} x_{e} \geq D_{i} \quad \forall i \in V \\
x_{e} \leq 1 \quad \forall e \in E \\
x_{e} \geq 0 \quad \forall e \in E \\
x_{e} \in \mathbb{Z}^{|E|}
\end{array}
$$

Here, $x_{e}$ is the indicator variable for the edges $e \in E . x_{e}=\mathbb{1}\{e \in A \subseteq E\}$. Which means, $x_{e}=1$, when $x_{e}$ is chosen in an outcome $A \subseteq E$.

The above formulation is an Integer Program of the combinatorial optimization problem. If the last condition (the integrality condition) is omitted, then the Integer problem will be relaxed to a Linear programming (LP) problem. We call this Linear programming as $L P_{B}$. If we find an optimum solution of the Linear Program which is integer then we can say that the integrality gap (discussed before) of that particular LP 
is 1 , and the optimum value of the LP then becomes the solution of IP.

We will prove that the corresponding LP of a bargaining game admits an integral solution if and only if the bargaining game has a stable outcome (as defined above). This means we could test the possibility of a stable solution of a bargaining game if we could find an optimum integral solution of its corresponding Linear Program. To arrive at this proof, we would depend on the Duality theory and complementary slackness condition of the LP.

Dual (We call it $L P_{B}^{[D]}$ ) of the above $\mathrm{LP}\left(L P_{B}\right)$ is as follows :

$$
\begin{array}{r}
\max \left(\sum_{i \in V} D_{i} z_{i}+\sum_{e \in E} y_{e}\right) \\
\sum_{i \in e} \rho_{i, e} z_{i}+y_{e} \leq C_{e} \quad \forall e \in E \\
z_{i} \geq 0 \quad \forall i \in V \\
y_{e} \leq 0 \quad \forall e \in E
\end{array}
$$

If the $\operatorname{LP}\left\{L P_{B}\right\}$ and the Dual $\left\{L P_{B}^{[D]}\right\}$ have feasible solution then for some specific feasible solution Complimentary Slackness conditions combining the $\operatorname{LP}\left\{L P_{B}\right\}$ and the Dual $\left\{L P_{B}^{[D]}\right\}$ are as follows :

$$
\begin{aligned}
\left(1-x_{e}\right) y_{e} & =0 \quad \forall e \in E \\
\left(\sum_{i \in e} \rho_{i, e} z_{i}+y_{e}-C_{e}\right) x_{e} & =0 \quad \forall e \in E \\
\left(\sum_{e \in \delta_{E}(i)} \rho_{i, e} x_{e}-D_{i}\right) z_{i} & =0 \quad \forall i \in V
\end{aligned}
$$

\subsubsection{Integrality from Stability}

Theorem 1. If a Bargaining Game has stable outcome then the corresponding Linear Program has integral optimum solution.

The theorem can be also restated in the following way:

If a Bargaining Game $B$ has stable outcome $F=\left\{A \subseteq E,\left\{S_{i, e}\right\}_{i \in V, e \in E}\right\}$ it is possible to construct an optimum solution $\left\{x_{e}\right\} \in \mathbf{N}, e \in E$ for $L P_{B}$

To prove the above mentioned theorem we are going to proceed the following way :

1. We will propose an Integer solution to the Linear program and a solution to its dual 
2. Using the stability condition of the given bargaining game we will prove certain conditions of the LP to show that our proposed solution is optimum.

3. First we are going to show that our proposed solution is feasible for the Linear Program.

4. Then we are going to show that our proposed solution is feasible for the Dual.

5. Next we would show that our proposed solutions satisfy complimentary slackness condition.

6. Then as per duality theory we could conclude that our proposed integer solution is optimum.

Proposed Solution :

$$
\begin{gathered}
\bar{x}_{e}=\left\{\begin{array}{ll}
1 & \text { if } e \in A \\
0 & \text { else }
\end{array} \quad \forall e \in E\right. \\
\bar{z}_{i}=\left\{\begin{array}{ll}
\max _{e \in \delta_{A}(i)}\left(\frac{S_{i, e}}{\rho_{i, e}}\right) & \text { if } \sum_{e \in \delta_{E}(i)} \rho_{i, e} x_{e}=D_{i} \\
0 & \text { else }
\end{array} \quad \forall i \in V\right. \\
\bar{y}_{e}=\left\{\begin{array}{ll}
C_{e}-\sum_{i \in e} z_{i} \rho_{i, e} & \text { if } e \in A \\
0 & \text { else }
\end{array} \quad \forall e \in E\right.
\end{gathered}
$$

Observation : The sum of satisfactions of an agent $i \in V$ in an outcome $A$ can be represented in terms of the indicator variable of LP:

$$
\begin{aligned}
\sum_{e \in \delta_{A}(i)} \rho_{i, e} & =\sum_{e \in \delta_{A}(i)} \rho_{i, e}+0 \\
& =\sum_{e \in \delta_{A}(i)} \rho_{i, e} \cdot 1+\sum_{e \in \delta_{E}(i) \backslash \delta_{A}(i)} \rho_{i, e} \cdot 0 \\
& =\sum_{e \in \delta_{A}(i)} \rho_{i, e} \cdot x_{e}+\sum_{e \in \delta_{E}(i) \backslash \delta_{A}(i)} \rho_{i, e} \cdot x_{e} \\
& =\sum_{e \in \delta_{E}(i)} \rho_{i, e} \bar{x}_{e}
\end{aligned}
$$

Lemma 1. If the Bargaining Game $B$ has stable outcome then the proposed solution $\left\{x_{e}\right\}_{e \in E}$ for $L P_{B}$ is feasible.

Proof. It is given that the Game $B$ has a Stable outcome, which means the Game $B$ satisfies the feasibility condition of the Bargaining Game, that means :

$$
\begin{aligned}
\sum_{e \in \delta_{A}(i)} \rho_{i, e} & \geq D_{i} \quad \forall i \in V \\
\rightarrow \sum_{e \in \delta_{E}(i)} \rho_{i, e} \bar{x}_{e} & \geq D_{i} \quad \forall i \in V
\end{aligned}
$$


Also by construction, $\bar{x}_{e} \in\{0,1\}$. So, the constraints $0 \leq \bar{x}_{e} \leq 1$ are satisfied

This proves all the constraints of $L P_{B}$ are satisfied, so $L P_{B}$ is feasible

Lemma 2. If the Bargaining Game B has stable outcome then the proposed solution $\left(\left\{z_{i}\right\}_{i \in V},\left\{y_{e}\right\}_{e \in E}\right)$ is feasible for the Dual $L P_{B}^{[D]}$.

Proof. We will first prove that our proposed solution satisfies the domain constraints of the indicator variables. For every Feasible outcome of the Game :

$$
\begin{gathered}
S_{i, e} \geq 0, \rho_{i, e}>0 \quad \forall i \in V, \forall e \in E \\
\text { so, } \max _{e \in \delta_{A}(i)}\left(\frac{S_{i, e}}{\rho_{i, e}}\right) \geq 0 \quad \forall i \in V \\
\text { so, } \bar{z}_{i} \geq 0 \quad \forall i \in V \quad\left[\because \bar{z}_{i}=\max _{e \in \delta_{A}(i)}\left(\frac{S_{i, e}}{\rho_{i, e}}\right)\right]
\end{gathered}
$$

Now we have to prove the non-positivity of the second variable of the Dual, i.e., $\bar{y}_{e} \leq 0 \quad \forall e \in E$.

To prove that we will separate the edges $e \in E$ into two partition set, $A$ and $E \backslash A$.

by construction, $\forall e \in A$ :

$$
\begin{aligned}
\bar{y}_{e} & =C_{e}-\sum_{i \in e} \bar{z}_{i} \rho_{i, e} \\
& =\sum_{i \in e} S_{i, e}-\sum_{i \in e} \bar{z}_{i} \rho_{i, e} \text { by stability condition }(3.1) \\
& =\sum_{i \in e}\left(S_{i, e}-\bar{z}_{i} \rho_{i, e}\right) \\
& =\sum_{i \in e} \rho_{i, e}\left(\frac{S_{i, e}}{\rho_{i, e}}-\bar{z}_{i}\right)
\end{aligned}
$$

It follows that

$$
\begin{aligned}
\max _{e \in \delta_{A}(i)}\left(\frac{S_{i, e}}{\rho_{i, e}}\right) & \geq \frac{S_{i, e}}{\rho_{i, e}} \forall i \in V \\
\rightarrow \bar{z}_{i} & \geq \frac{S_{i, e}}{\rho_{i, e}} ; \forall i \in V \quad\left[\because \text { by construction } \bar{z}_{i}=\max _{e \in \delta_{A}(i)}\left(\frac{S_{i, e}}{\rho_{i, e}}\right)\right] \\
\rightarrow 0 & \geq \frac{S_{i, e}}{\rho_{i, e}}-\bar{z}_{i} \quad \forall i \in V \\
\rightarrow 0 & \geq \sum_{i \in e}\left(\frac{S_{i, e}}{\rho_{i, e}}-\bar{z}_{i}\right) \quad \forall e \in A\left[\because \bar{z}_{i}, S_{i, e}, \rho_{i, e} \geq 0\right] \\
\rightarrow 0 & \geq \sum_{i \in e} \rho_{i, e}\left(\frac{S_{i, e}}{\rho_{i, e}}-\bar{z}_{i}\right) \quad \forall e \in A \quad \because \rho_{i, e}>0 \\
\rightarrow 0 & \geq \bar{y}_{e} ; \forall e \in A[U \operatorname{Using} \text { Eqn.3.7] }
\end{aligned}
$$


Also by construction, $\bar{y}_{e}=0 \quad \forall e \in E \backslash A$.

Combining both cases $A$ and $E \backslash A$

$$
\begin{aligned}
y_{e} \leq 0 \quad \forall e \in A \cup(E \backslash A) \\
\therefore y_{e} \leq 0 \quad \forall e \in E
\end{aligned}
$$

Now we have proven that the constraints pertaining to the domain of the dual variables are satisfied. Next we are going to prove the first constraints of the Dual, i.e., $\sum_{i \in e} \rho_{i, e} z_{i}-y_{e} \leq C_{e} \forall e \in E$ are satisfied.

To prove that, again we will separate all the edges $e \in E$ into two partition sets, $A$ and $E \backslash A$

Case $e \in A$ : by construction, $\forall e \in A$ :

$$
\begin{aligned}
\bar{y}_{e} & =C_{e}-\sum_{i \in e} \rho_{i, e} \bar{z}_{i} \\
\rightarrow \sum_{i \in e} \rho_{i, e} \bar{z}_{i}+\bar{y}_{e} & =C_{e} \quad e \in A .
\end{aligned}
$$

Case $e \in E \backslash A$ : we have to analyze this using the Stability condition. From stability we get

$$
\begin{aligned}
\sum_{i \in f} \max _{e \in \delta_{A}(i)}\left(\frac{S_{i, e}}{\rho_{i, e}}\right) \rho_{i, f} & \leq C_{f} \quad \forall f \in E \backslash A \\
\rightarrow \sum_{i \in f} \bar{z}_{i} * \rho_{i, f} & \leq C_{f} \quad \forall f \in E \backslash A \\
\rightarrow \sum_{i \in f} \rho_{i, f} \bar{z}_{i}+\bar{y}_{e} & \leq C_{f} \quad \forall f \in E \backslash A \quad\left[\because \bar{y}_{e}=0 \quad \forall e \in E \backslash A\right]
\end{aligned}
$$

So combining the cases $\forall e \in A$ and $\forall e \in E \backslash A$ we can conclude. $\sum_{i \in e} \rho_{i, f} \bar{z}_{i}+\bar{y}_{e} \leq C_{e} \quad \forall e \in E$.

So all the feasibility criteria are satisfied. Now we need to check the Complimentary slackness conditions to prove that our proposed solution is optimal solution.

Lemma 3. If Bargaining Game Problem B has stable outcome then Complimentary Slackness (3.3), (3.4), (3.5) are satisfied with our proposed solution.

Proof. To prove the different Complimentary Slackness conditions, we will proceed by partitioning the sets of players and edges. For the first two conditions which are applicable for all the edges, we will separate the edges $e \in E$ into two partition sets $A$ and $E \backslash A$. For the last condition which is applicable for all the vertices, we will separate the vertices $e \in E$ into two partition sets of oversaturated and tight set.

Complimentary Slackness Case $1(3.3)$ : 
For all edges chosen in the outcome, by construction $x_{e}=1$ and for all edges not chosen in the outcome, by construction $y_{e}=0$. Combining these two cases it follows $\left(1-x_{e}\right) y_{e}=0 \quad \forall e \in E$.

Complimentary Slackness Case 2 (3.4) :

For all edges not chosen in the outcome, by construction $x_{e}=0$ and for all edges chosen in the outcome, by construction $y_{e}=C_{e}-\sum_{i \in e} \rho_{i, e} z_{i}$. So combining it follows that

$\left(\sum_{i \in e} \rho_{i, e} z_{i}+y_{e}-C_{e}\right) x_{e}=0 \quad \forall e \in E$.

Complimentary Slackness Case $3(3.5)$ :

It follows that $\sum_{e \in \delta_{A}(i)} \rho_{i, e}=\sum_{e \in \delta_{E}(i)} \rho_{i, e}$. From Stability condition we know $\left(\sum_{e \in \delta_{A}(i)} \rho_{i, e}-D_{i}\right) S_{i, e}=$ $0, \forall i \in V, \quad \forall e \in E$, now, partition the players into sets of oversaturated agents and tight agents. It is obvious that for the tight agents where $\sum_{e \in \delta_{E}(i)} \rho_{i, e} x_{e}=D_{i}$ complimentary slackness condition (3.5) $\left(\sum_{e \in \delta_{E}(i)} \rho_{i, e} x_{e}-D_{i}\right) z_{i}=0$ automatically holds. For all over saturated agents where, $\sum_{e \in \delta_{E}(i)} \rho_{i, e} x_{e}>D_{i}$, the payment is always 0, i.e., $S_{i, e}=0$; therefore $\frac{S_{i, e}}{\rho_{i, e}}=0$; hence, $\max _{e \in \delta_{A}(i)}\left(\frac{S_{i, e}}{\rho_{i, e}}\right)=0$ which means $z_{i}=0$.

Thus complimentary slackness condition (3.5) $\left(\sum_{e \in \delta_{E}(i)} \rho_{i, e} x_{e}-D_{i}\right) z_{i}=0 \quad \forall i \in V$ is satisfied for all the agents,

We proved that the LP and its dual are feasible and all the complimentary slackness conditions are satisfied with our proposed integral solution, if we use the Stability condition. So our proposed integral solution is optimal.

\subsubsection{Stability from Integrality}

Theorem 2. If the corresponding linear program of a bargaining game has integer optimum solution then there exists a stable outcome for that bargaining game.

Let us consider that the corresponding Linear Program $L P_{B}$ of a bargaining game $B$ has an integer optimum solution $\left\{\overline{x_{e}}\right\} \in\{0,1\}, \forall e \in E$. By strong duality, the dual $L P_{B}^{D}$ of the LP would also have an optimum solution, say those are $\overline{z_{i}}, \overline{y_{e}} \in \mathbb{R}, \forall i \in V, \forall e \in E$. We are going to propose an outcome of $B$ and with that, we would show that the proposed outcome satisfies our defined stability condition. To arrive at that condition, we have to prove different lemmas on the way.

Lemma 4. There should be at least one tight agent in every edge within an optimum solution of the corresponding combinatorial optimization problem of any bargaining game.

Proof. For the sake of contradiction let us assume that all the agents $i \in \bar{e}$ of a chosen edge $\bar{e} \in\left\{e: \bar{x}_{e}=1\right\}$ in the optimum solution $\left\{\bar{x}_{e}\right\}$ of $L P_{B}$ of the corresponding bargaining game $B$ are oversaturated. Say agent $i \in \bar{e}$ has demand $D_{i}$ and receives total satisfaction $\sum_{e \in E: i \in e} \rho_{i, e} \bar{x}_{e}$. Because we assumed that each of the agents are oversaturated then $\sum_{e \in E: i \in e} \rho_{i, e} \bar{x}_{e}>D_{i}, \forall i \in \bar{e}$ So $\sum_{e \in E \backslash \bar{e}: i \in e} \rho_{i, e} \bar{x}_{e}+\rho_{i, \bar{e}} \bar{x}_{\bar{e}}>D_{i}, \forall i \in \bar{e}$. So 
we can decrease value of the indicator variable $\bar{x}_{\bar{e}}$ by a small amount $\epsilon>0$. Yet the demand for all the oversaturated agents in that agent would be still satisfied. That would mean the solution that we got can be improved (minimized). So that means the solution that was given is not an optimal solution, which contradicts the given condition. So not all the agents in a chosen edge can be oversaturated.

Lemma 5. Given an optimum solution $\left\{\overline{x_{e}}\right\}_{e \in E}$ of $L P_{B}$ and $\left(\left\{\bar{z}_{i}\right\}_{i \in V},\left\{\bar{y}_{e}\right\}_{e \in E}\right)$ of the dual LP $D$ of the Bargaining Game B, there exists a set of distributions $\left\{\theta_{i, e}\right\}_{i \in V}, \forall e \in E$ which satisfies the condition $\overline{z_{i}} \rho_{i, e}+$ $\theta_{i, e} \overline{y_{e}} \geq 0$

Proof. Here we are going to propose values for the distribution $\theta_{i, e}$. For that we are going to propose separate values of $\theta_{i, e}$ for oversaturated and tight agents. Because $\left\{\theta_{i, e}\right\}_{i \in V, e \in E}$ is a distribution over all the players $i \in e$ for a selected edge $e \in E: \bar{x}_{e}>0$, our proposed value of $\theta_{i, e}$ should satisfy the following condition :

1. $\theta_{i, e} \in \mathbb{R}_{+}$

2. $\sum_{i \in e} \theta_{i, e}=1, \forall e \in E: \bar{x}_{e}>0$

Proposed values for the distribution :

Let us consider $\theta_{i, e}=0$ for all the oversaturated agents. So, $\left(\sum_{f \in \delta_{A}(i)} \rho_{i, f}-D_{i}\right) \theta_{i, e}=0, \forall e \in A, A \subseteq E, A=\left\{e: \bar{x}_{e}>0\right\}$

We have already seen that there should be at least one tight agent. So if there is only one tight agent we can put all the weight of the distributions to that agent for that edge and this way the conditions $\sum_{i \in e} \theta_{i, e}=1$ will always hold true, with our proposed solution.

Now we propose to choose the distribution factor for all the tight agents $i \in e^{T} \subseteq e: \sum_{f \in \delta_{A}(i)} \rho_{i, f}=D_{i}$ of an edge $e \in E$, in the following way:

$$
\theta_{i, e}=\frac{\rho_{i, e} \overline{z_{i}}}{\sum_{i \in e^{T}} \rho_{i, e} \overline{z_{i}}}
$$

As the solutions of the LP and the Dual are optimum, the complimentary slackness is satisfied. From 
complimentary slackness (2) it follows $\forall e \in E: x_{e}>0$

$$
\begin{aligned}
\sum_{i \in e} \rho_{i, e} \overline{z_{i}}+\overline{y_{e}} & =C_{e} \\
\rightarrow \sum_{i \in e} \rho_{i, e} \overline{z_{i}}+\overline{y_{e}} & >0\left[\because C_{e}>0\right] \\
\rightarrow \sum_{i \in e} \rho_{i, e} \overline{z_{i}} & >0\left[\because y_{e} \leq 0\right] \\
\rightarrow \sum_{i \in e^{T}} \rho_{i, e} \bar{z}_{i}+\sum_{i \in e \backslash e^{T}} \rho_{i, e} \overline{z_{i}} & >0[\text { dividing into tight and over satuared agents }] \\
\rightarrow \sum_{i \in e^{T}} \rho_{i, e} \overline{z_{i}}+\sum_{i \in e \backslash e^{T}} \rho_{i, e} \cdot 0 & >0\left[\overline{z_{i}}=0 \forall \text { oversatuared agents }\right] \\
\rightarrow \sum_{i \in e^{T}} \rho_{i, e} \bar{z}_{i} & >0
\end{aligned}
$$

So the denominator of proposed distribution would be always positive. So the distribution is a valid number. Now, $\rho_{i, e}, \overline{z_{i}} \geq 0$, So $\theta_{i, e} \geq 0$, and also it follows that

$$
\sum_{i \in e} \theta_{i, e}=\sum_{i \in e} \frac{\rho_{i, e} \overline{z_{i}}}{\sum_{i \in e^{T}} \rho_{i, e} \overline{z_{i}}}=\frac{\sum_{i \in e} \rho_{i, e} \overline{z_{i}}}{\sum_{i \in e^{T}} \rho_{i, e} \bar{z}_{i}}=\frac{\sum_{i \in e^{T}} \rho_{i, e} \overline{z_{i}}+\sum_{i \in e \backslash e^{T}} \rho_{i, e} \overline{z_{i}}}{\sum_{i \in e^{T}} \rho_{i, e} \overline{z_{i}}}=\frac{\sum_{i \in e^{T}} \rho_{i, e} \overline{z_{i}}+0}{\sum_{i \in e^{T}} \rho_{i, e} \overline{z_{i}}}=1
$$

So our proposed $\theta_{i, e}$ is found to be a valid distribution. Now we can prove the lemma (5).

$$
\begin{aligned}
\overline{z_{i}} \rho_{i, e}+\theta_{i, e} \overline{y_{e}} & =\overline{z_{i}} \rho_{i, e}+\frac{\rho_{i, e} \overline{z_{i}}}{\sum_{i \in e^{T}} \rho_{i, e} \overline{z_{i}}} \overline{y_{e}} \\
& =\overline{z_{i}} \rho_{i, e}\left(1+\frac{\overline{y_{e}}}{\sum_{i \in e^{T}} \rho_{i, e} \overline{z_{i}}}\right) \\
& =\overline{z_{i}} \rho_{i, e}\left(1+\frac{\overline{y_{e}}}{\sum_{i \in e^{T}} \rho_{i, e} \overline{z_{i}}+\sum_{i \in e \backslash e^{T}} \rho_{i, e} \overline{z_{i}}}\right) \quad\left[\overline{z_{i}}=0 \forall \text { oversatuared agents }\right] \\
& =\overline{z_{i}} \rho_{i, e}\left(1+\frac{\overline{y_{e}}}{\sum_{i \in e} \rho_{i, e} \overline{z_{i}}}\right) \\
& =\overline{z_{i}} \rho_{i, e}\left(\frac{\sum_{i \in e} \rho_{i, e} \overline{z_{i}}+\overline{y_{e}}}{\sum_{i \in e} \rho_{i, e} \overline{z_{i}}}\right) \\
& =\bar{z}_{i} \rho_{i, e}\left(\frac{C_{e}}{\sum_{i \in e} \rho_{i, e} \overline{z_{i}}}\right) \quad\left[\sum_{i \in e} \rho_{i, e} \overline{z_{i}}+\overline{y_{e}}=C_{e} \text { from complimentary slackness }(3)\right]
\end{aligned}
$$

Now $\overline{z_{i}} \geq 0, \rho_{i, e} \geq 0, C_{e}>0$. So $\overline{z_{i}} \rho_{i, e}+\theta_{i, e} \overline{y_{e}} \geq 0$

Now with the help of these two lemmas we are going to prove the theorem. Our approach for proving the theorem would be to first propose an outcome of the bargaining game and then we are going to show that the proposed outcome is feasible and stable. We already know that the bargaining game outcome consists of two parts. One is the set of edges that are selected and other is the set of payments for the vertices and for 
the edges. The chosen set of edges are already given to us in the optimum solution. So set of chosen edges $A \subseteq E$ are given by $A=\left\{e: x_{e}=1\right\}$. So we are going to only propose the payments.

Proposed Bargaining Game Outcome (Payments) : We are going to express our proposed payments as a linear combination of the two dual variables $\overline{z_{i}}$ and $\overline{y_{e}}$. So our payment is going to be summation of two parts. One part $z_{i}$ would signify payment (possibly excess than the required amount) which is a characteristic of vertex $i \in e$ and another part $y_{e}$ would signify discount (negative payment), which is the characteristics of the edge $e \in A$ itself. For that we are going to define a discount distribution factor $\theta_{i, e}$, which distributes the edge discount to all the participating members. This above mentioned discount distribution factor $\theta_{i, e}$ would satisfy

1. $\theta_{i, e} \in \mathbb{R}_{+}$.

2. $\sum_{i \in e} \theta_{i, e}=1 \forall e \in A$.

3. $\theta_{i, e} \geq-\frac{z_{i}}{y_{e}} \rho_{i, e} \forall e \in A, \forall i \in e$.

4. $\left(\sum_{f \in \delta_{A}(i)} \rho_{i, f}-D_{i}\right) \theta_{i, e}=0, \forall e \in A$.

We have already shown in our previous lemma that it is always possible to construct such $\theta_{i, e}$ which satisfies all the conditions shown above.

Now our proposed solution for payment distribution is

$$
\bar{S}_{i, e}=\left\{\begin{array}{ll}
\rho_{i, e} \bar{z}_{i} & \text { if } x_{e}=1 \\
0 & \text { else }
\end{array}\right\}+\theta_{i, e} y_{e}, \quad \forall e \in E, \quad \forall i \in V
$$

This can be written in a more simplified form :

$$
\bar{S}_{i, e}=\rho_{i, e} \overline{z_{i}} \overline{x_{e}}+\theta_{i, e} \overline{y_{e}} \quad \forall e \in E \quad \forall i \in V
$$

From the third condition of the definition of $\theta_{i, e}$ it follows that $\bar{S}_{i, e} \geq 0$.

From Complimentary slackness condition (3.3) it follows that $\overline{y_{e}}=0$ if $\overline{x_{e}}=0$, so by construction we have $\bar{S}_{i, e}=0$ if $\overline{x_{e}}=0$; also $\theta_{i, e} \overline{y_{e}}=0$ if $\overline{x_{e}}=0$. This indicates that no one is paying any amount for the unchosen edges. 
From Complimentary slackness condition (3.4) it follows that $\forall e \in E$, if $\overline{x_{e}}=1$,

$$
\begin{aligned}
\sum_{i \in e} \rho_{i, e} \overline{z_{i}}+\overline{y_{e}} & =C_{e} \\
\rightarrow \sum_{i \in e} \rho_{i, e} \overline{z_{i}}+\overline{y_{e}} \sum_{i \in e} \theta_{i, e} & =C_{e} \quad\left[\because \rightarrow \sum_{i \in e} \theta_{i, e}=1\right] \\
\rightarrow \sum_{i \in e} \rho_{i, e} \overline{z_{i}}+\sum_{i \in e} \theta_{i, e} \overline{y_{e}} & =C_{e} \\
\rightarrow \sum_{i \in e}\left(\rho_{i, e} \overline{z_{i}}+\theta_{i, e} \overline{y_{e}}\right) & =C_{e} \\
\rightarrow \sum_{i \in e} \overline{S_{i, e}} & =C_{e}
\end{aligned}
$$

That means only the participating agents are completely paying for the services, which signifies that no one is paying for the service that they are not part of. This is one of the condition of Stability which is satisfied.

From Complimentary slackness condition (3.5) it follows that if $\sum_{e \in \delta_{E}(i)} \rho_{i, e} \bar{x}_{e}>D_{i}$ then $\overline{z_{i}}=0$ also from construction if $\sum_{e \in \delta_{E}(i)} \rho_{i, e} \overline{x_{e}}>D_{i}$ then $\forall e \in A \quad \theta_{i, e}=0$. That means if $\sum_{e \in \delta_{E}(i)} \rho_{i, e} \overline{x_{e}}>D_{i}$ then $\rho_{i, e} \overline{z_{i}}+\theta_{i, e} \overline{y_{e}}=0$ or $\overline{S_{i, e}}=0$. This is another one of the condition of Stability which is satisfied.

Consider an unselected edge $e \in \delta_{E}(i) \backslash \delta_{A}(i)$, so $\overline{y_{e}}=0$.

The solution is feasible, hence

$$
\begin{aligned}
\overline{y_{e}} & \leq 0 \quad \forall e \in E \\
\rightarrow \theta_{i, e} \overline{y_{e}} & \leq 0 \quad \forall e \in E \quad \because \theta_{i, e} \geq 0 ; \forall e \in E \forall i \in V \\
\rightarrow \overline{S_{i, e}}-\rho_{i, e} \overline{S_{i, e}} & \leq 0 \quad \forall e \in E \forall i \in V \\
\rightarrow \overline{S_{i, e}} & \leq \rho_{i, e} \overline{z_{i}} \forall e \in E \forall i \in V \\
\rightarrow \frac{S_{i, e}}{\rho_{i, e}} & \leq \overline{z_{i}} ; \quad \forall e \in E \forall i \in V \\
\rightarrow \max _{e \in \delta_{E}(i)}\left(\frac{\overline{S_{i, e}}}{\rho_{i, e}}\right) & \leq \overline{z_{i}} \forall i \in V \\
\rightarrow \max _{e \in \delta_{A}(i)}\left(\frac{S_{i, e}}{\rho_{i, e}}\right) & \leq \overline{z_{i}} \forall i \in V \quad \because \overline{S_{i, e}}=0 \forall e \notin A \\
\rightarrow \max _{e \in \delta_{A}(i)}\left(\frac{\overline{S_{i, e}}}{\overline{\rho_{i, e}}}\right) \rho_{i, f} & \leq \rho_{i, f} \overline{z_{i}} \forall i \in V \forall f \in E \\
\rightarrow \sum_{i \in f} \max _{e \in \delta_{A}(i)}\left(\frac{\overline{S_{i, e}}}{\rho_{i, e}}\right) \rho_{i, f} & \leq \sum_{i \in f} \rho_{i, f} \overline{z_{i}} \forall i \in V \forall f \in E
\end{aligned}
$$


From the feasibility of the Dual it follows that

$$
\begin{aligned}
\sum_{i \in e} \rho_{i, e} \overline{z_{i}}+\overline{y_{e}} & \leq C_{e} \forall e \in E \\
\rightarrow \sum_{i \in f} \rho_{i, f} \overline{z_{i}} & \leq C_{f} \forall f \notin A \quad \because y_{f}=0 \quad \forall f \notin A
\end{aligned}
$$

Combining this we get

$$
\sum_{i \in f} \max _{e \in \delta_{A}(i)}\left(\frac{\overline{S_{i, e}}}{\rho_{i, e}}\right) \rho_{i, f} \leq C_{f} \forall f \notin A \quad \because y_{f}=0 \quad \forall f \notin A
$$

This indicates that the final Stability condition is also satisfied. Since, all of the stability conditions are satisfied, proposed solution would be stable.

Theorem 3. The Bargaining Game Problem B has stable outcome $F=\left\{A \subseteq E,\left\{S_{i, e}\right\}_{i \in V, e \in E}\right\}$ if and only if the corresponding Linear Program $L P_{B}$ has integral optimum solution.

Combining Theorems 1 and 2 we can conclude that the above theorem is true.

\subsection{Stability for socially aware agents}

The Saturation concept, which was discussed before, influences the stability considerably. Our present definition of stability may not be enough to study many practical cases. Let us understand the limitations of our present stability definition with a simple practical example.

Example 4.5.1. Consider a simple bargaining game with three players $A, B, C$ where each has one unit demand. So $D_{A}=D_{B}=D_{C}=1$. Each player can form two services, one service with each of the other players. Consider the services are denoted by $A B, B C, C A$ and each service costs unit amount. So $C_{A B}=C_{B C}=C_{C A}=1$. Say each service gives unit satisfaction to each player. That means, $\rho_{A, A B}=\rho_{A, C A}=\rho_{B, A B}=\rho_{B, B C}=\rho_{C, C A}=\rho_{C, B C}=1$.

This game can be depicted with a simple triangular (cyclic) graph with three nodes and three edges. (Fig 4.3)

It follows that, this bargaining game has a feasible outcome only if one of the players gets 2 services. That means at least one of the players would be over-saturated. Assume that player $B$ takes 2 services $A B, B C$ and she doesn't pay anything for those services. Then $A$ and $C$ can form a service between them and then $B$ would be left unsatisfied. This situation is clearly not stable. The solution is feasible, but not stable.

It is interesting to note, that the relationship between the stability of a bargaining game outcome with the integrality gap of its underlying linear optimization problem can detect the instability of those special situations. For example, the underlying LP of the above mentioned bargaining game on a triangle where the demands, satisfactions and the costs are of unit amount (Fig 3), will have the integral solution as 


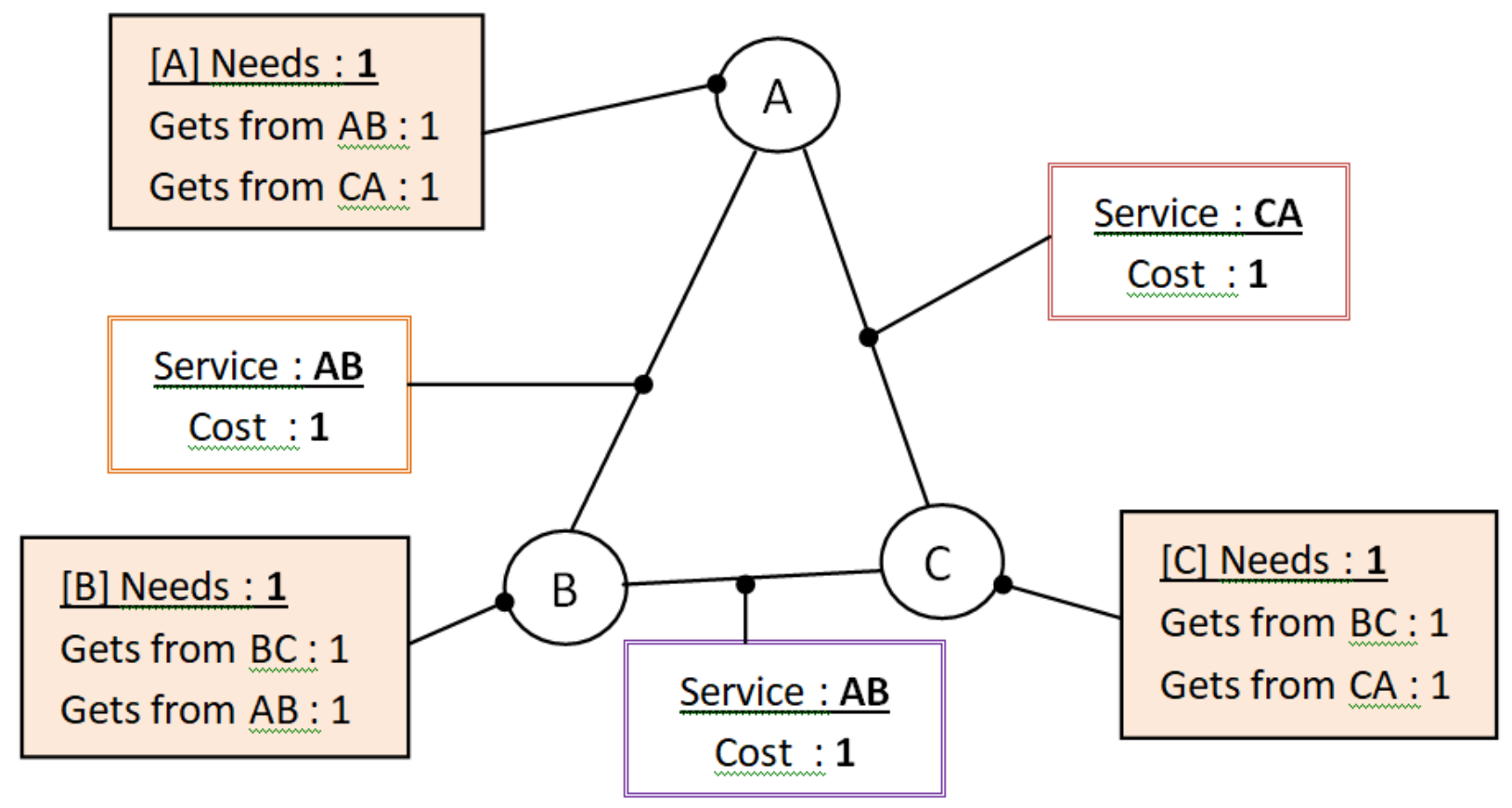

Figure 4.3: Bargaining Game Example with Odd Cycle.

$x_{A B}+x_{B C}+x_{C A}=1+1+0=2$, whereas, its linear optimum solution is $x_{A B}+x_{B C}+x_{C A}=\frac{1}{2}+\frac{1}{2}+\frac{1}{2}=\frac{3}{2}<2$. So the integrality gap is given by $\frac{3 / 2}{2}=\frac{3}{4}$, which is not 1 . That means the underlying integral optimum solution and the linear optimum solution of the bargaining game would not coincide, which indicates that any solution would be unstable.

The instability situation that we have seen in the above example, can be found in many different practical scenarios. It can be found out that in many places, where our definition of stability would be too strict. If we generalize these situations, then we could say there might be some extra conditions within a network, over our proposed stability conditions, that the players need to satisfy to make the outcome stable.

So, we need to revise our stability conditions to incorporate those above mentioned situations. We need to relax our notion and allow the players to pay for the services, they are not part of or if they are oversaturated. But how much extra they would be willing to pay is the question and we should find a formulation for that as well. Intuitively we should suggest two different payments for all the players. One part is the payment which are independent of any special situation, that may arise for the player for being in a special group. And another payment is only for those special situations. 


\subsubsection{Critical Constraints}

Here we are going to formally define the general critical constraints which are important for a new version of stability.

Definition 4.5.1. A critical constraint $K$ of a bargaining game $B=\left(G=(V, E), C_{e}, D_{i}, \rho_{i, e}\right)$ is defined as a triplet $K=\left(H \subseteq E, \alpha_{H} \in \mathbb{Q}_{++},\left\{\sigma_{e} \in \mathbb{Q}_{++}\right\}_{e \in H}\right)$, such that for the chosen edges $A \subseteq E$ of any feasible outcome the following conditions are satisfied : $\sum_{e \in H \cap A} \sigma_{e} \geq \alpha_{H}$.

Example 4.5.2. If we consider the above mentioned bargaining game, on a triangle, where the demands, satisfactions and the costs are of unit amount (Fig 4.3), critical constraint would be $R=\{A B, B C, C A\}, \alpha_{H}=$ $2, \sigma_{A B}=\sigma_{B C}=\sigma_{C A}=1$. In fact, for all vertex-cover type bargaining games, for every odd cycles of edges $C \subseteq E$ should have $\alpha_{C}=\frac{|C|+1}{2}$

In this context we define the term $\Gamma=\left\{\left(H_{i}, \alpha_{H_{i}},\left\{\sigma_{e}\right\}_{e \in H_{i}}\right)\right\}_{i \in\{1, . ., k\}}$, which indicates a set of critical constraints within the bargaining game $B$. Also $V\left(H_{j}\right)=\cup_{i \in H_{j}} i$.

\subsubsection{Combinatorial Optimization with the New Constraints}

With this new concept of critical constraints the underlying linear program would also change. The Linear Program $\left(L P_{B}\right)$ would have an extra set of constraints for each of the critical constraints. These constraints are redundant for the Integer program, as they are guaranteed to be satisfied if the solution for the linear program is integer. This way the extra constraints can be thought of as the cutting planes of the linear program. The extra constraint set would be as follows:

$$
\sum_{e \in H \cap A} \sigma_{e} x_{e} \geq \alpha_{H} \quad \forall H \in \Gamma
$$

Denote this new linear program with $L P_{(B, \Gamma)}$, The complete formulation of $L P_{(B, \Gamma)}$ is as follows:

$$
\begin{array}{r}
\min \sum_{e \in E} C_{e} x_{e} \\
\sum_{e \in \delta_{E}(i)} \rho_{i, e} x_{e} \geq D_{i} \quad \forall i \in V \\
\sum_{e \in H \cap A} \sigma_{e} x_{e} \geq \alpha_{H} \quad \forall H \in \Gamma \\
x_{e} \leq 1 \quad \forall e \in E \\
x_{e} \geq 0 \quad \forall e \in E
\end{array}
$$

\subsubsection{Relaxed Notion of Stability}

To define the stability in the new context of critical constraint, we need to first understand the notion that if some agents are part of a critical constraint within a network, then some agents might need to pay for the 
services they have not taken. In addition to that, if an agent is within a critical constraint and oversaturated, then she still may have to pay some amount for the services within the critical constraint group.

We can try to understand that with the help of our previous example (Fig 3.3). In this example, we have to choose at least 2 services. Consider service $A B, B C$ is chosen. But all three agents $A, B, C$ should pay something for each of those two services. Otherwise any other set of two services could be chosen, which will make the situation unstable.

Now we have agreed that all the agents within a constraint group should share some responsibility of the payment for the services within that constraint to satisfy the demand of the constraints. Still, the question remains, how much each of the agent should pay, so that they don't feel deprived.

To approach the formulation of payment for each agent, we should divide the payment in two parts. One is independent of the critical constraints and the other part is for being part of different critical constraint group. So our augmented definition of stability considering the critical constraints are given as follows :

Definition 4.5.2. A feasible outcome $A \subseteq E$ of bargaining game $B$, with set of critical constraints $\Gamma$ would be called $\Gamma$-stable if the outcome satisfies the following conditions :

1. The payments for every agent $i \in V$ for every edge $e \in E$ are given by $S_{i, e}=S_{i, e}^{\emptyset}+\sum_{j \in \Gamma: e \in H_{j}} S_{i, e}^{H_{j}}$. $S_{i, e}^{\emptyset}$ indicates the payment for the agent $i \in V$ for edge $e \in E$ independent of any critical constraint. $S_{i, e}^{H_{j}}$ indicates the payment for the agent $i \in V$ for edge $e \in E$ within a critical constraint. Both $S_{i, e}^{\emptyset}$ and $S_{i, e}^{H_{j}}$ are non-negative.

2. For payment, independent of any critical constraint, agent pays none for the services they are not part of : $S_{i, e}^{\emptyset}=0 \quad \forall e \notin \delta_{A}(i)$.

3. For payment, independent of any critical constraint, oversaturated agents pays nothing: $S_{i, e}=0 \quad \forall e \in$ $\delta_{A}(i): \sum_{e \in \delta_{A}(i)} \rho_{i, e}>D_{i}$.

4. Payment for any critical constraint is positive only when the demand for critical constraint is tightly satisfied, else it would be $0: S_{i, e}^{H_{j}}=0, \quad \forall e \in \delta_{A}(i): \sum_{e \in H_{j} \cap A} \sigma_{e}>\alpha_{j}$

5. Current combined option is the best option : $\forall f \in E \backslash A$

$\sum_{j \in f} \max _{e \in \delta_{A}(j)}\left(\frac{S_{j, e}}{\rho_{j, e}}\right) \rho_{j, f}+\sum_{t \in f \cap H_{j}, H_{j} \in \Gamma} \max _{e \in \delta_{A \cap H_{j}}(t)}\left(\frac{\sigma_{f}}{\sigma_{e}}\right) \sum_{H_{j} \in \Gamma: e \in H_{j}} S_{i, e}^{H_{j}} \leq C_{f}$.

From the second condition it follows that :

$$
\left(\sum_{e \in \delta_{A}(i)} \rho_{i, e}-D_{i}\right) S_{i, e}^{\emptyset}=0 \quad \forall i \in V \quad \forall e \in E
$$




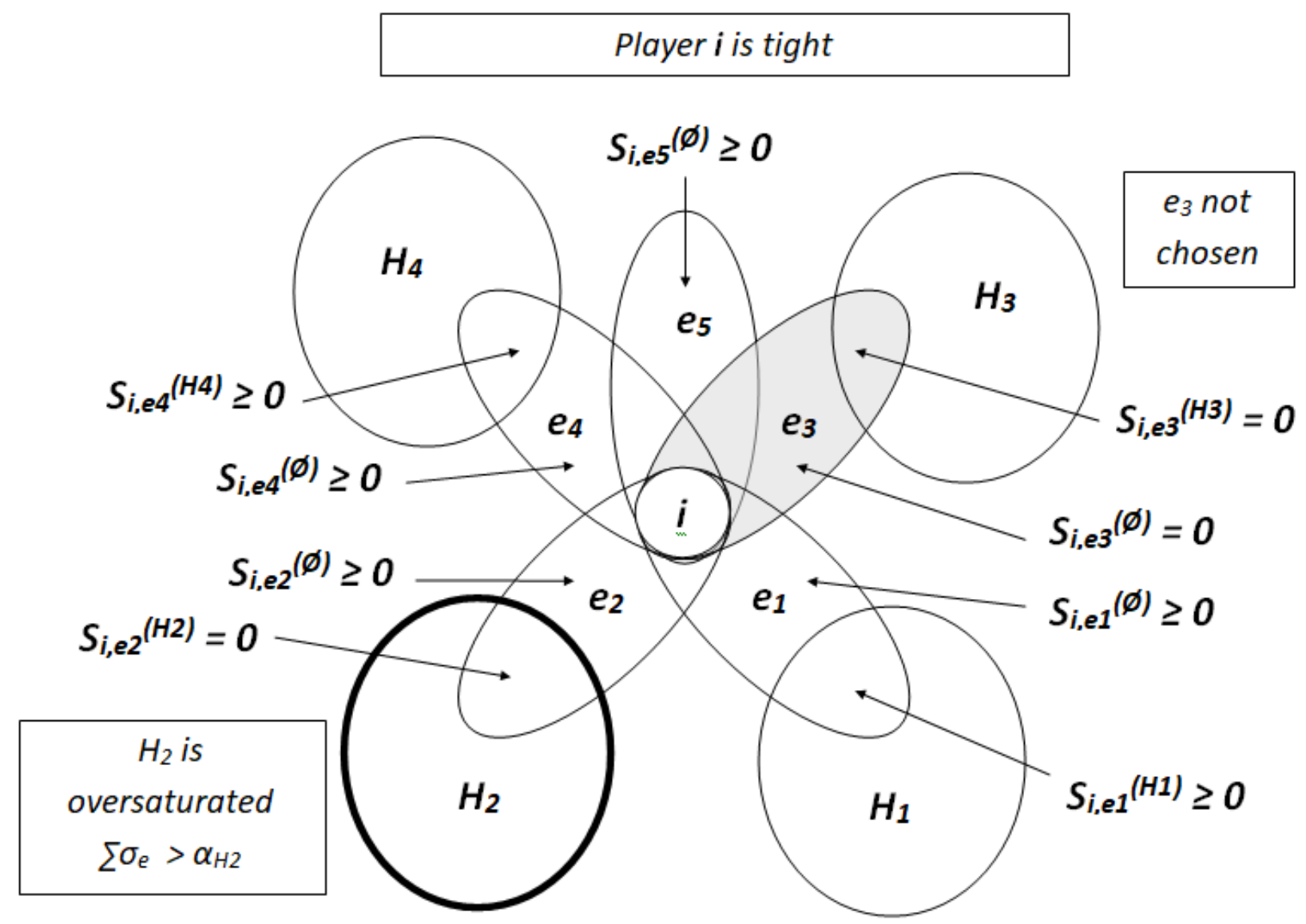

Figure 4.4: Payment distribution with critical constraints for tight agent

In this context, we define two terms called $\Gamma$-Stability and $\emptyset$-Stability. $\emptyset$-Stability defines the stability conditions which were defined in the definition 3.2.2. $\Gamma$-Stability defines the stability conditions which were defined in the definition 3.4.2.

\subsubsection{Augmented Representation of the Bargaining Game}

If we add more agents to the original bargaining game and add extra agents to some of its edges, the data structure (data model) of the bargaining game won't change. Same way the structure of the corresponding combinatorial optimization problem would also not change.

So, if we treat each critical constraints as a virtual agent, then it is possible to represent the bargaining game and its corresponding combinatorial optimization with the original Linear program formulation. Now, lets formally define the bargaining game $B$ with the critical constraints as virtual agents. This revised definition of bargaining game can be termed as augmented bargaining game $B^{\prime}$. 


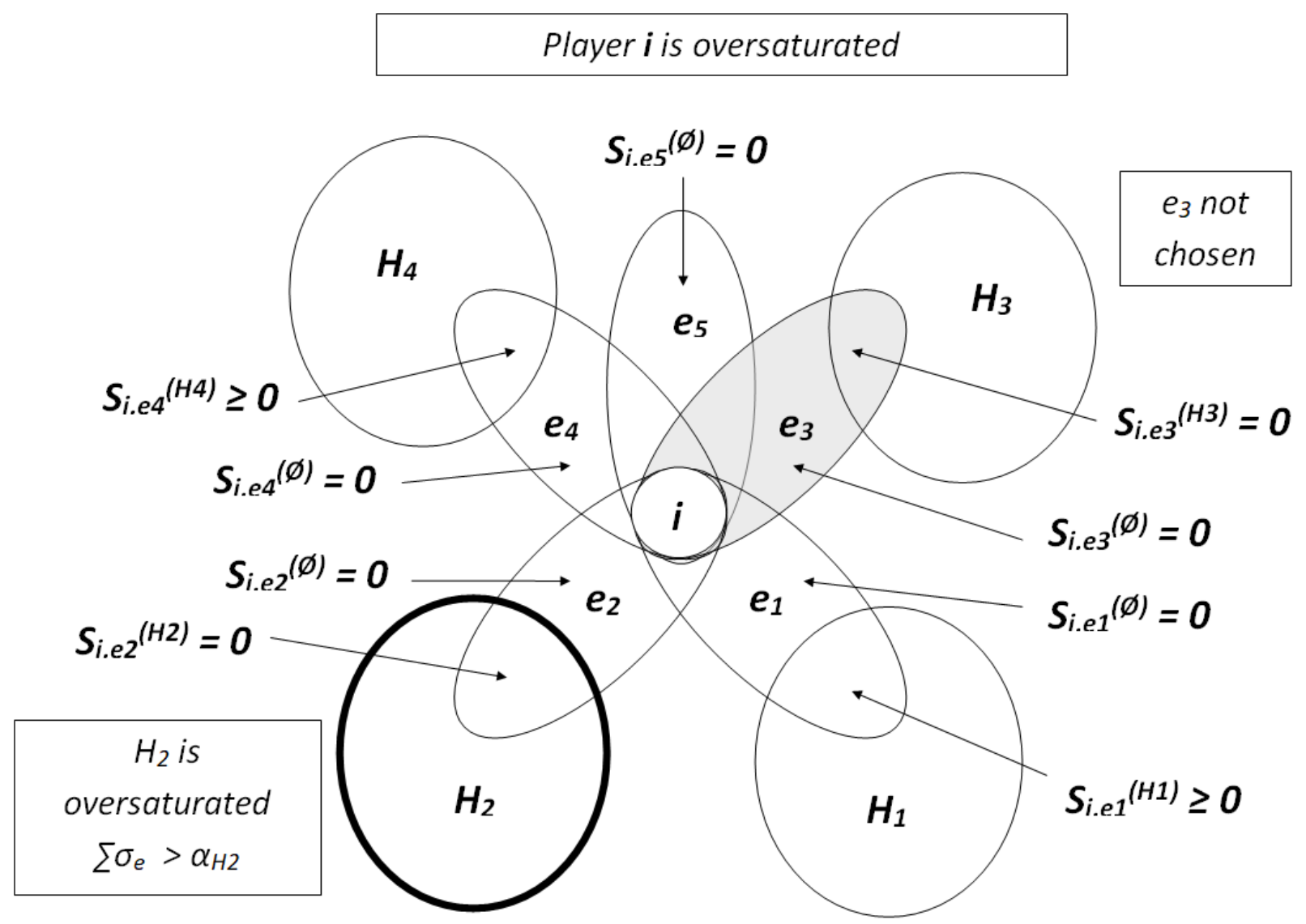

Figure 4.5: Payment distribution with critical constraints for oversaturated agent

Definition 4.5.3. Augmented bargaining game $B^{\prime}$ of bargaining game $B$ with critical constraints $\Gamma$ is described by the following components:

1. Hypergraph $G^{\prime}=\left(V^{\prime}, E^{\prime}\right)$, where augmented set of nodes are $V^{\prime}=V \cup\{n+1, \ldots ., n+k\}$. Here the no. of constraints $k=|\Gamma|$ and index variable $n+j$ for constraint $j \in\{1, \ldots, k\}$, where $V=$ $\{1, \ldots, n\}, n \in \mathbf{N}$. Each augmented edge $e^{\prime}$ is given by differently for two different cases. The edges those are not part of any critical constraints they remain unchanged. The edges which are part of the same critical constraint, will have an extra virtual member fir them. So the augmented set of edges are $E^{\prime}=\left(\left\{e^{\prime}=e \cup(n+j): e \in H_{j}\right\}_{H_{j} \in \Gamma} \cup\left\{e: e \in E \backslash \cup_{H_{j} \in \Gamma} H_{j}\right\}\right)$. This way it follows that the augmented set of edge has bijective relation with the original set of edge.

2. Edge weights $\left\{C_{e^{\prime}}\right\}_{e^{\prime} \in E}=\left\{C_{e}\right\}_{e \in E}$.

3. Vertex weights $\left\{D_{i}^{\prime}\right\}_{i \in V^{\prime}}=\left\{D_{i}\right\}_{i \in V} \cup\left\{\alpha_{j}\right\}_{j \in\{1, \ldots, k\}}$.

4. Satisfaction of every player from every service 


$$
\left\{\rho_{i, e}^{\prime}\right\}_{i \in V^{\prime}, e^{\prime} \in E}=\left(\left\{\rho_{i, e} \in \mathbf{Q}_{++}\right\}_{e \in E, i \in V}\right) \cup\left(\left\{\sigma_{e^{\prime}}\right\}_{e^{\prime} \in \cup_{H_{j} \in \Gamma} H_{j}, j \in\{1, \ldots, k\}}\right) .
$$

In more descriptive way, the augmented bargaining game is formed such that every constraint can be thought of as an extra virtual player that is added to the original set of agents. The number of edges remain same as the original number of edges, but each of the edges, which are part of a constraint, will have an extra agent in it. That extra agent is the virtual agent for the said constraint. The cost for each edge will be unchanged. The demand will be unchanged for the original bargaining game. But the virtual agents will have the demands same as the demand of the constraint. The satisfaction for every original player remains unchanged. Each virtual player represents a critical constraint, which is composed of some edges. Each virtual player belongs to all those edges and it gets the satisfaction from all those edges.

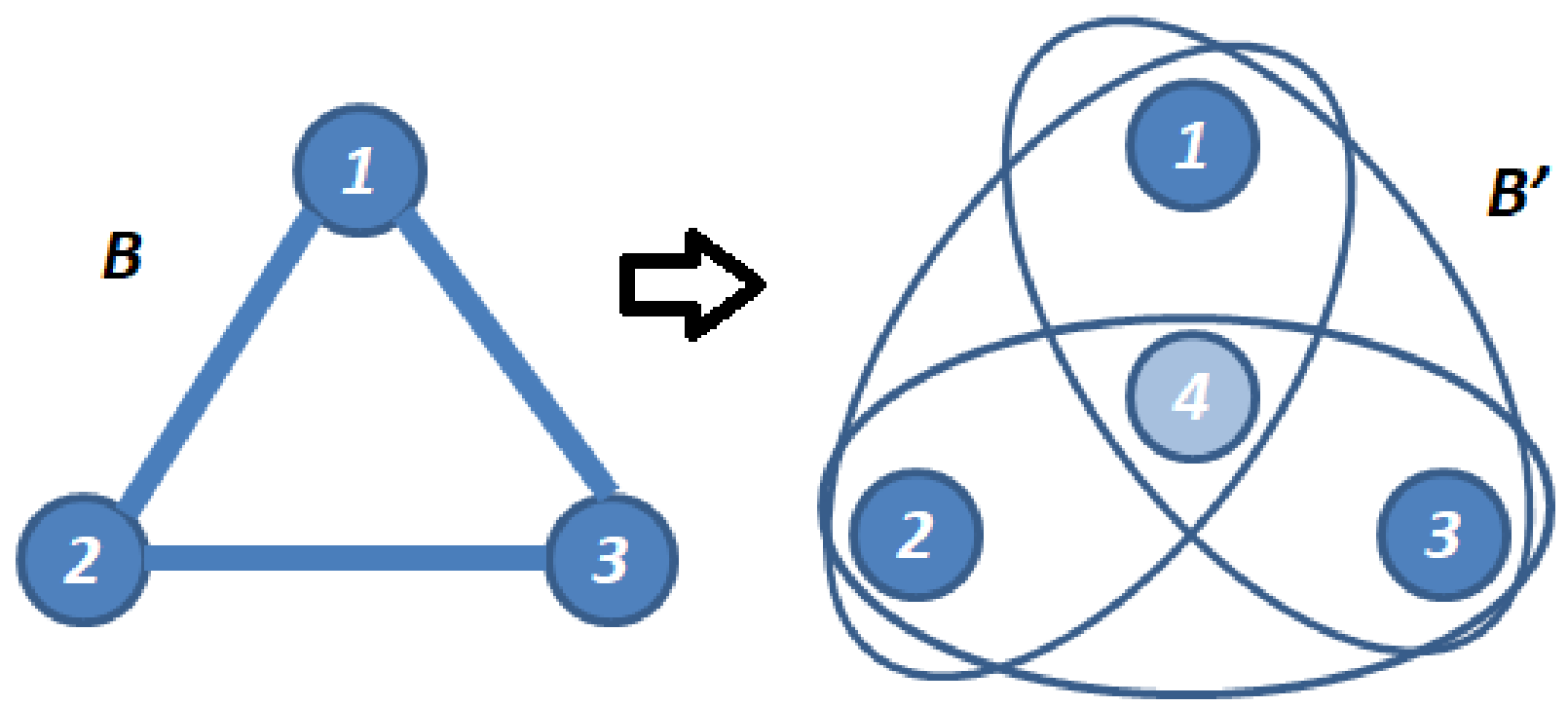

Figure 4.6: Augmented representation of the game with critical constraints

Example 4.5.3. In fig. 4.6, the bargaining game $B$ has three vertices $1,2,3$ and edges $\{1,2\},\{2,3\},\{3,1\}$. $B$ has one critical constraint $k$ which comprises of the edges $\{1,2\},\{2,3\},\{3,1\}$. The bargaining game $B$ can be represented by an augmented bargaining game $B^{\prime}$, where there is an extra virtual vertex 4 , which represents the constraint $k$. In this augmented bargaining game each of the edge get an extra vertex 4 in them. So the augmented edges become $\{1,2,4\},\{2,3,4\},\{3,1,4\}$. The augmented game has no critical constraint. The critical constraint $k$ is now represented by the extra vertex 4 , and the condition of the critical constraint is represented by the demand of 4 in this new augmented game.

\subsubsection{Augmented Game and the Revised Combinatorial Optimization Problem}

Lemma 6. Augmented bargaining game $B^{\prime}$ of original $B$ with $\Gamma$ critical constraints, admits an $\emptyset$ stable outcome if and only if the integrality gap of $L P_{(B, \Gamma)}$ is 1. 
This lemma (can be cast as a Corollary) follows as the original $L P_{B}$ formulation is valid for any hypergraphs. So in the augmented game $B^{\prime}$, it is just another hypergraph where there are some extra vertices and some edges with one extra vertex in them. So its corresponding $L P_{B^{\prime}}$ has the same data structure as $L P_{(B, \Gamma)}$ (Type of constraints are same, only number of constraints are more, because of extra number of virtual vertices). So bargaining game $B^{\prime}$ admits a stable outcome as defined in definition 3.2.2 ( $\emptyset$ stable) if and only if the integrality gap of $L P_{B^{\prime}}$ is 1 , i.e. the integrality gap of is $L P_{(B, \Gamma)}$ is 1 .

\subsubsection{Relationship of the new Stability with Integrality Gap}

If we use the augmented representation of a Bargaining game and find that the augmented game is stable as per the stability definition 3.2.2 then we say that the augmented game is $\emptyset$-Stable, which means all the feasibility conditions of the bargaining game are satisfied. In addition to that, the demands of the virtual agents are also satisfied; this fact means all the critical constraints, which are represented as the demands of the corresponding virtual agents, are also satisfied. Now because the augmented game is stable, any agent, be it actual agent or virtual, is not paying for the service she is not part of. In addition to that, any virtual oversaturated agent is not paying for any of the services.

Lemma 7. If $B$ admits a $\Gamma$-stable outcome then $B^{\prime}$ admits an $\emptyset$-stable outcome.

Proof. It is given that the original game $B$ has a $\Gamma$-stable outcome. Let us consider that $\Gamma$-stable outcome $F$ is given by $F=\left(A \subseteq E,\left\{S_{i, e}\right\}_{e \in E, i \in V}\right)$. By definition of $\Gamma$-stability, for every agent $i \in V$ for every edge $e \in E$ there exists non-negative payment $S_{i, e}=S_{i, e}^{\emptyset}+\sum_{j \in \Gamma: e \in H_{j}} S_{i, e}^{H_{j}}$, where $S_{i, e}^{\emptyset}, S_{i, e}^{H_{j}} \geq 0$.

Correspondingly, we define the outcome of the augmented game $B^{\prime}$. We set $A=A^{\prime}$ and $S_{i, e}^{\prime}=S_{i, e}^{\emptyset}$; This is possible because there is bijection between $E$ and $E^{\prime}$. For each critical constraint $H_{j} \in \Gamma$, we set $S_{n+j, e}^{\prime}=\sum_{i \in V\left(H_{j}\right)} S_{i, e}^{H_{j}}$.

With this, first we are going to prove that our proposed outcome of the augmented game $B^{\prime}$ is feasible. After that we are going to prove that the outcome is stable as per the original definition of stability.

To prove that our proposed outcome of the augmented game $B^{\prime}$ is feasible, we first make sure that the demands are satisfied. $A$ satisfies Demand Satisfaction for $B$. That means the demands of all the nodes in $V$ are all satisfied. At the same time, the chosen services in a stable (that means also feasible) outcome also guarantee to satisfy any critical constraint (see Definition 3). That means all the nodes in $V^{\prime} \backslash V$ are also satisfied. Hence, $A=A^{\prime}$ satisfies Demand Satisfaction for $B^{\prime}$. Next we show the cost recovery conditions 
are also satisfied. for all $e \in A$

$$
\begin{aligned}
\sum_{i \in V} S_{i, e^{\prime}}^{\prime} & =\sum_{i \in V} S_{i, e^{\prime}}+\sum_{j \in[k]} S_{n+j, e^{\prime}}^{H_{j}} \\
& =\sum_{i \in V} S_{i, e}^{\emptyset}+\sum_{j \in[k]} \sum_{i \in V\left(H_{j}\right)} S_{n+j, e^{\prime}}^{H_{j}} \\
& =\sum_{i \in V} S_{i, e}^{\emptyset}+\sum_{j \in[k]}\left(\sum_{i \in V\left(H_{j}\right)} S_{n+j, e^{\prime}}^{H_{j}}+0\right) \\
& =\sum_{i \in V} S_{i, e}^{\emptyset}+\sum_{j \in[k]}\left(\sum_{i \in V\left(H_{j}\right)} S_{n+j, e^{\prime}}^{H_{j}}+\sum_{i \notin V\left(H_{j}\right)} S_{n+j, e^{\prime}}^{H_{j}}\right)\left[\because S_{n+j, e^{\prime}}^{H_{j}}=0, \forall i \notin V\left(H_{j}\right)\right] \\
& =\sum_{i \in V} S_{i, e}^{\emptyset}+\sum_{j \in[k]} \sum_{i \in V} S_{n+j, e^{\prime}}^{H_{j}}=\sum_{i \in V}\left(S_{i, e}^{\emptyset}+\sum_{j \in[k]} S_{n+j, e^{\prime}}^{H_{j}}\right) \\
& =\sum_{i \in V} S_{i, e}=C_{e}
\end{aligned}
$$

This proves that the cost recovery criteria of feasibility is also satisfied. Hence the proposed outcome of the augmented game $B^{\prime}$ is feasible.

Next we are going to prove the $\emptyset$-stability (original stability definition) of the augmented game $B^{\prime}$. First we check the property that agents only pay for the services they are part of. From the definition of $\Gamma$-stability it is clear that agents pay only when they are members of an actual chosen service, or they are part of a group. When they are part of a critical constraint group, by definition they are members of the edges of that group in the augmented game. So it is clear that for the augmented game $B^{\prime}$ players are not paying for the services that they are not part of.

Next we are going to prove that only tight players pay positive amounts for their services. For $i \in V^{\prime}, e \in E^{\prime}$, let us consider two partitioned sets of the augmented players, viz, $V \& V^{\prime} \backslash V$.

Case 1 : As per the $\Gamma$-stability of $B$, if $i \in V$ and $S_{i, e}^{\emptyset}>0$, then player $i$ is tight, i.e, $S_{i, e^{\prime}}^{\prime}>0$. That means in augmented game $B^{\prime}$, if any player $i \in V$ pays a positive amount, then player $i$ is tight.

Case 2 : If $i \in V^{\prime} \backslash V$, i.e., $i=n+j, j \in[k]$ and $\sum_{j \in[k]} S_{i, e^{\prime}}^{H_{j}}>0$, then the demand of constraint group $H_{j}$ is tightly satisfied, as per the definition of $\Gamma$-stability. That means virtual player $i \in V^{\prime} \backslash V$ is tight. Now as per our proposed solution $S_{i, e^{\prime}}^{\prime}=\sum_{j \in[k]} S_{i, e^{\prime}}^{H_{j}}$. So $\sum_{j \in[k]} S_{i, e^{\prime}}^{H_{j}}>0$ means $S_{i, e^{\prime}}^{\prime}>0$. That means in augmented game $B^{\prime}$, if any player $i \in V^{\prime} \backslash V$ pays a positive amount, then the player $i$ is tight.

Combining these two cases we can conclude, that $\forall i \in V^{\prime}$ if $S_{i, e^{\prime}}^{\prime}>0$ then the player $i$ is tight. This is another condition of stability. 
Finally we need to prove that the chosen options are the best options for all the players. Let us consider an edge $f^{\prime} \in A^{\prime}$. Let us consider two partition sets $U \subseteq V$ and $W \subseteq[k]$ of $f^{\prime}$, i.e, $f^{\prime}=U \cup W, U \cap W=\emptyset$, now

$$
\begin{aligned}
\sum_{t \in f^{\prime}} \max _{e^{\prime} \in \delta_{A^{\prime}}(t)}\left(\frac{S_{t, e^{\prime}}^{\prime}}{\rho_{t, e^{\prime}}}\right) \rho_{t, e^{\prime}} & =\sum_{i \in U} \max _{e^{\prime} \in \delta_{A^{\prime}}(i)}\left(\frac{S_{i, e^{\prime}}^{\prime}}{\rho_{i, e^{\prime}}}\right) \rho_{i, e^{\prime}}+\sum_{j \in W} \max _{e^{\prime} \in \delta_{H_{j}}(t)}\left(\frac{S_{n+j, e^{\prime}}^{\prime}}{\sigma_{j, e^{\prime}}}\right) \sigma_{j, e^{\prime}} \\
& =\sum_{i \in U} \max _{e^{\prime} \in \delta_{A^{\prime}}(i)}\left(\frac{S_{i, e^{\prime}}^{\emptyset}}{\rho_{i, e^{\prime}}}\right) \rho_{i, e^{\prime}}+\sum_{j \in W} \max _{e^{\prime} \in \delta_{H_{j}}(t)}\left(\frac{\sigma_{j, e^{\prime}}}{\sigma_{j, e^{\prime}}}\right) \sum_{t \in[k]} S_{t, e^{\prime}}^{H_{j}} \\
& \geq C_{f}[B y \Gamma-\text { stability }] \\
& =C_{f^{\prime}}
\end{aligned}
$$

So combining all these we can conclude that $B^{\prime}$ is stable.

Lemma 8. If $B^{\prime}$ admits an $\emptyset$-stable outcome then $B$ admits an $\Gamma$-stable outcome.

Proof. Consider stable outcome $F^{\prime}=\left(A^{\prime} \subseteq E^{\prime},\left\{S_{i, e^{\prime}}^{\prime}\right\}_{e^{\prime} \in E^{\prime}, i \in V^{\prime}}\right)$ of $B^{\prime}$. For each critical constraint $j \in[k]$, let us consider a distribution $\theta_{i, j} \in \mathbb{R}_{++}$over agents $i \in V\left(H_{j}\right)$, which means $\sum_{i \in V\left(H_{j}\right)} \theta_{i, j}=1$. We also explicitly set $\theta_{i, j}=0$, if $i \notin V\left(H_{j}\right)$. Next let us define the outcome of $B$ as $F=\left(A \subseteq E,\left\{S_{i, e}\right\}_{e \in E, i \in V}\right)$; We first set $A=A^{\prime}$, and because of bijection between $E$ and $E^{\prime}$ this is possible. Now we set, $\forall i \in V, e \in E, S_{i, e}^{\emptyset}=S_{i, e^{\prime}}^{\prime}$, $\forall j \in[k], S_{i, e}^{H_{j}}=\theta_{i, j} \cdot S_{n+j, e^{\prime}}^{\prime}$, This way $S_{i, e}=S_{i, e}^{\emptyset}+\sum_{e \in H_{j} \in \Gamma} S_{i, e}^{H_{j}}=S_{i, e^{\prime}}^{\prime}+\sum_{e \in H_{j} \in \Gamma} \theta_{i, j} \cdot S_{n+j, e^{\prime}}^{\prime}$

Next we show that $B$ is feasible. Because, $B^{\prime}$ is stable, it is also feasible. By definition of feasibility, $B^{\prime}$ satisfies the demands of all the agents. That means, the demands of all the actual players and all the virtual players, i.e, demands of the critical constraints are satisfied. Hence $A \subseteq E$ satisfies demand satisfaction for $B$. Now, we are going to show another condition of feasibility, i,e. condition cost recovery. Because 
$B^{\prime}$ is stable it satisfies all cost recovery condition, So $\forall e \in A$ of $B$,

$$
\begin{aligned}
C_{e} & =\sum_{i \in V^{\prime}} S_{i, e^{\prime}}^{\prime}=\sum_{i \in V} S_{i, e^{\prime}}+\sum_{i \in V^{\prime} \backslash V} S_{i, e^{\prime}} \\
& =\sum_{i \in V} S_{i, e^{\prime}}^{\prime}+\sum_{j \in[k]} S_{n+j, e^{\prime}}^{\prime} \\
& =\sum_{i \in V} S_{i, e^{\prime}}^{\prime}+\sum_{j \in[k]} S_{n+j, e^{\prime}}^{\prime} \cdot \sum_{i \in V\left(H_{j}\right)} \theta_{i, j} \\
& =\sum_{i \in V} S_{i, e^{\prime}}^{\prime}+\sum_{j \in[k]} S_{n+j, e^{\prime}}^{\prime} \cdot\left(\sum_{i \in V\left(H_{j}\right)} \theta_{i, j}+\sum_{i \in V \backslash V\left(H_{j}\right)} \theta_{i, j}\right) \\
& =\sum_{i \in V} S_{i, e^{\prime}}^{\prime}+\sum_{j \in[k]} S_{n+j, e^{\prime}}^{\prime} \cdot \sum_{i \in V} \theta_{i, j} \\
& =\sum_{i \in V}\left(S_{i, e^{\prime}}^{\prime}+\sum_{j \in[k]} S_{n+j, e^{\prime}}^{\prime} \theta_{i, j}\right) \\
& =\sum_{i \in V}\left(S_{i, e}^{\emptyset}+\sum_{j \in[k]} S_{i, e}^{H_{j}}\right)=\sum_{i \in V} S_{i, e} .
\end{aligned}
$$

This proves the cost recovery condition is satisfied for all edges in $F$. So $F$ is feasible.

Now we are going to prove that $F$ is $\Gamma$-Stable. For this we are going to show that each of the condition defined in the definition of $\Gamma$-Stability are satisfied. The first two conditions are obvious from the construction. Next we prove the third, fourth and fifth conditions, one by one.

First we check that only the tight agents pay positive amounts for an actual edge. We know that $\forall i \in V, e \in E$, where $S_{i, e}^{\emptyset}>0$ the corresponding $S_{i, e^{\prime}}^{\prime}>0$, but because $B^{\prime}$ is stable, all $i \in V^{\prime}$ are tight. So all agents in $F$, who pay positive amounts, independent of any constraint, are tight.

Here we define a concept called collective tightness, which indicates whether the requirements or demands of a critical constraints are satisfied tightly or not. Next we check the collective tightness of the critical constraints. Let us consider $i \in V ; e \in E, j \in[k]$ so that $S_{i, e}^{H_{j}}>0$, which also means $\theta_{i, e} \cdot S_{n+j, e^{\prime}}^{\prime}>0$ or $\theta_{i, e}>0$ and $S_{n+j, e^{\prime}}^{\prime}>0$, as $\theta_{i, e}$ is a distribution over $V\left(H_{j}\right)$, so $\theta_{i, e}>0$, indicates $i \in V\left(H_{j}\right)$ and $S_{n+j, e^{\prime}}^{\prime}>0$ indicates $e^{\prime} \in A^{\prime} \cap H_{j}$. Now since $F^{\prime}$ is stable, the constraint $H_{j}$ is satisfied tightly. That means $F$ satisfies the collective tightness of the critical constraints.

Finally we have to show that collectively the current option is the best option. For that let's consider an edge $f \notin A$, which means $f^{\prime} \notin A^{\prime}$. The edge $f$ consists of some vertices which are denoted by $i_{1}, \ldots, i_{l}$, so $f=\left\{i_{1}, \ldots, i_{l}\right\}$ and $f$ is part of some critical constraints $\left\{j_{1}, \ldots, i_{m}\right\}$. Now, let us consider an arbitrary 
$e_{j} \in \delta_{A}(j)$ and $e_{t} \in A \cap H_{j}$, which automatically means $e_{j}, e_{t} \in A^{\prime}$

$$
\begin{aligned}
\sum_{r=1}^{l}\left(\frac{S_{i_{r}, e_{j}}^{\emptyset}}{\rho_{i_{r}, e_{r}}}\right) \rho_{i_{r}, f}+\sum_{t=1}^{m}\left(\frac{\sigma_{j_{t}, f}}{\sigma_{j_{t}, e_{t}}}\right) \sum_{t \in[k]} S_{t, e_{t}}^{H_{j_{t}}} & =\sum_{r=1}^{l}\left(\frac{S_{i_{r}, e_{j}}^{\prime}}{\rho_{i_{r}, e_{r}}}\right) \rho_{i_{r}, f}+\sum_{t=1}^{m}\left(\frac{\sigma_{j_{t}, f}}{\sigma_{j_{t}, e_{t}}}\right) \sum_{t \in[k]} \theta_{i, e} S_{n+j_{t}, e^{\prime}}^{\prime} \\
& =\sum_{r=1}^{l}\left(\frac{S_{i_{r}, e_{j}}^{\prime}}{\rho_{i_{r}, e_{r}}}\right) \rho_{i_{r}, f}+\sum_{t=1}^{m}\left(\frac{\sigma_{j_{t}, f}}{\sigma_{j_{t}, e_{t}}}\right) S_{n+j_{t}, e^{\prime}} \sum_{t \in[k]} \theta_{i, e} \\
& =\sum_{r=1}^{l}\left(\frac{S_{i_{r}, e_{j}}^{\prime}}{\rho_{i_{r}, e_{r}}}\right) \rho_{i_{r}, f}+\sum_{t=1}^{m}\left(\frac{\sigma_{j_{t}, f}}{\sigma_{j_{t}, e_{t}}}\right) S_{n+j_{t}, e^{\prime}}^{\prime} \\
& \leq C_{f}\left[\because F^{\prime} \text { is stable }\right]
\end{aligned}
$$

So this means for all the players including the virtual players, the current option is found to be the best option. That means the original game $B$ is $\Gamma$-stable

We have proved the conditions needed for the games with critical constraints to have a stable solution. Now, we can prove the following theorem.

Theorem 4. $B^{\prime}$ admits an $\emptyset$-stable outcome if and only if $B$ admits an $\Gamma$-stable outcome.

Proof. The theorem automatically follows from the previous two lemmas. 


\section{Chapter 5}

\section{Possible Future Work}

In our work we have studied different notions of stability both from a strict context and from the context of socially aware agents. In this process we have extended our study by generalizing many different aspects of bargaining games. We conducted our study of bargaining game as a covering problem, in contrast to the widely used packing type problems. In our work we answered some of the questions related to the stability of bargaining games, but also opened up the possibilities of many other research questions.

The relaxed notion of stability that we introduced can be further studied with experimental data. Many statistical exercises or machine learning work can be carried out to study approximately stable solutions for bargaining games. These type of questions are mainly in the domain of Economists and Social scientists. If we only confine our thinking to the theoretical and mathematical problems surrounding to our work, then there are many interesting questions as well. Some of them are as described below.

In our work we only considered linear relaxations. If we extend our thought then the natural question would be: Is there a natural stability notion associated with semidefinite programming relaxations (or in general non-linear relaxations)? Is there any implication on the stability notion if the starting linear program relaxation is tightened by any of the well-studied lift-and-project systems, for example Lovasz-Schrijver [23], Sherali-Adams [29], or Lasserre [22] ? We have studied the case where the integrality gap is 1, but is there a natural stability notion associated with convex relaxations in which the integrality gap is not 1, yet bounded? 


\section{Bibliography}

[1] Yossi Azar, Benjamin E. Birnbaum, Elisa Celis, Nikhil R. Devanur, and Yuval Peres. Convergence of local dynamics to balanced outcomes in exchange networks. In 50th Annual IEEE Symposium on Foundations of Computer Science, FOCS 2009, October 25-27, 2009, Atlanta, Georgia, USA, pages 293-302. IEEE Computer Society, 2009.

[2] MohammadHossein Bateni, MohammadTaghi Hajiaghayi, Nicole Immorlica, and Hamid Mahini. The cooperative game theory foundations of network bargaining games. In International Colloquium on Automata, Languages, and Programming, pages 67-78. Springer, 2010.

[3] Mohsen Bayati, Christian Borgs, Jennifer Chayes, Yash Kanoria, and Andrea Montanari. Bargaining dynamics in exchange networks. Journal of Economic Theory, 156:417-454, 2015.

[4] Adrian Bock, Karthekeyan Chandrasekaran, Jochen Könemann, Britta Peis, and Laura Sanità. Finding small stabilizers for unstable graphs. Mathematical Programming, 154(1-2):173-196, 2015.

[5] Elisa Celis, Nikhil R. Devanur, and Yuval Peres. Local dynamics in bargaining networks via random-turn games. In Internet and Network Economics - 6th International Workshop, WINE 2010, Stanford, CA, USA, December 13-17, 2010. Proceedings, volume 6484 of Lecture Notes in Computer Science, pages 133-144. Springer, 2010.

[6] Tanmoy Chakraborty and Michael Kearns. Bargaining solutions in a social network. In Internet and Network Economics, 4th International Workshop, WINE 2008, Shanghai, China, December 17-20, 2008. Proceedings, pages 548-555, 2008.

[7] Tanmoy Chakraborty, Michael Kearns, and Sanjeev Khanna. Network bargaining: algorithms and structural results. In Proceedings 10th ACM Conference on Electronic Commerce (EC-2009), Stanford, California, USA, July 6-10, 2009, pages 159-168, 2009.

[8] Georgios Chalkiadakis, Edith Elkind, and Michael Wooldridge. Computational Aspects of Cooperative Game Theory. Synthesis Lectures on Artificial Intelligence and Machine Learning, 16. Morgan \& Claypool Publishers, Williston, VT, 2012. 
[9] T-H Hubert Chan, Fei Chen, and Li Ning. Optimizing social welfare for network bargaining games in the face of unstability, greed and spite. In European Symposium on Algorithms, pages 265-276. Springer, 2012 .

[10] Karen S. Cook, Richard M. Emerson, Mary R. Gillmore, and Toshio Yamagishi. Distribution of power in exchange networks: Theory and experimental results. American Journal of Sociology, pages 275-305, 1983.

[11] X. Deng and Q. Fang. Algorithmic cooperative game theory. In Pareto Optimality, Game Theory And Equilibria, Springer Optimization and Its Applications, Volume 17, pages 159-185. Springer, 2008.

[12] X. Deng, T. Ibaraki, and H. Nagamochi. Algorithmic aspects of the core of combinatorial optimization games. mor, 24(3):751-766, 1999.

[13] Moez Draief and Milan Vojnović. Bargaining dynamics in exchange networks. In 2010 48th Annual Allerton Conference on Communication, Control, and Computing (Allerton), pages 1303-1310. IEEE, 2010.

[14] Linda Farczadi, Konstantinos Georgiou, and Jochen Könemann. Stable marriage with general preferences - extended abstract. In Algorithmic Game Theory - 7th International Symposium, SAGT 2014, Haifa, Israel, September 30 - October 2, 2014. Proceedings, pages 25-36, 2014.

[15] Konstantinos Georgiou, George Karakostas, Jochen Könemann, and Zuzanna Stamirowska. Social exchange networks with distant bargaining. Theoretical Computer Science, 554:263-274, 2014.

[16] Daniel Granot and Frieda Granot. On some network flow games. Mathematics of Operations Research, 17(4):792-841, 1992.

[17] B. Guenin, J. Konemann, and L. Tuncel. A Gentle Introduction to Optimization.

[18] MohammadTaghi Hajiaghayi and Hamid Mahini. Bargaining Networks, pages 1-5. Springer US, Boston, MA, 2014.

[19] Takehiro Ito, Naonori Kakimura, Naoyuki Kamiyama, Yusuke Kobayashi, and Yoshio Okamoto. Efficient stabilization of cooperative matching games. Theoretical Computer Science, 677:69-82, 2017.

[20] Yashodhan Kanoria, Mohsen Bayati, Christian Borgs, Jennifer Chayes, and Andrea Montanari. A natural dynamics for bargaining on exchange networks. arXiv preprint arXiv:0911.1767, 2009.

[21] Jon M. Kleinberg and Éva Tardos. Balanced outcomes in social exchange networks. In Proceedings of the 40th Annual ACM Symposium on Theory of Computing, Victoria, British Columbia, Canada, May 17-20, 2008, pages 295-304. ACM, 2008.

[22] Jean B. Lasserre. An explicit exact SDP relaxation for nonlinear 0-1 programs. In IPCO'01: Proceedings of the 8th International Conference on Integer Programming and Combinatorial Optimization, volume 2081 of Lecture Notes in Comput. Sci., pages 293-303. Springer, Berlin, 2001. 
[23] László Lovász and Alexander Schrijver. Cones of matrices and set-functions and 0-1 optimization. SIAM Journal on Optimization, 1(2):166-190, May 1991.

[24] J. Nash. The bargaining problem. Econometrica, 18:155-162, 1950.

[25] Martin J. Osborne and Ariel Rubinstein. A Course in Game Theory.

[26] Sharon C. Rochford. Symmetrically pairwise-bargained allocations in an assignment market. Journal of Economic Theory, 34(2):262-281, 1984.

[27] Ariel Rubinstein. Perfect equilibrium in a bargaining model. Econometrica: Journal of the Econometric Society, pages 97-109, 1982.

[28] Lloyd S. Shapley and Martin Shubik. The assignment game. I. The core. International Journal of Game Theory, 1(2):111-130, 1972.

[29] Hanif D. Sherali and Warren P. Adams. A hierarchy of relaxations between the continuous and convex hull representations for zero-one programming problems. SIAM J. Discrete Math., 3(3):411-430, 1990.

[30] David Willer. Network Exchange Theory. Praeger Publishers, 1999. 